\title{
ERROR ESTIMATES FOR A CLASS OF FINITE DIFFERENCE-QUADRATURE SCHEMES FOR FULLY NONLINEAR DEGENERATE PARABOLIC INTEGRO-PDES
}

\author{
I. H. BISWAS, E. R. JAKOBSEN, AND K. H. KARLSEN
}

\begin{abstract}
Error estimates are derived for a class of monotone finite differencequadrature schemes approximating viscosity solutions of nonlinear degenerate parabolic integro-PDEs with variable diffusion coefficients. The relevant equations can be viewed as Bellman equations associated to a class of controlled jump-diffusion (Lévy) processes. Our results cover both finite and infinite activity cases.
\end{abstract}

\section{INTRODUCTION}

In this article we consider error estimates for finite difference type numerical schemes for degenerate and fully nonlinear parabolic integro-partial differential equations (integro-PDEs henceforth) of Bellman type. We write the equation in the following abstract form,

$$
u_{t}(t, x)+F\left(t, x, u(t, x), D u(t, x), D^{2} u(t, x), u(t, \cdot)\right)=0 \text { in } Q_{T}
$$

where $T>0$ is a constant and $Q_{T}=[0, T) \times \mathbb{R}^{d}$ and we impose a terminal condition,

$$
u(T, x)=u_{0}(x) \text { for all } x \in \mathbb{R}^{d} .
$$

The nonlocal feature of the equation is indicated by the term $u(t, \cdot)$. For any $(t, x, r, p, X) \in \mathbb{R} \times \mathbb{R}^{d} \times \mathbb{R} \times \mathbb{R}^{d} \times \mathbb{S}^{d}$ and for any 'sufficiently well behaved' $\varphi$, the nonlinearity $F$ is defined as follows

$$
\begin{aligned}
& F(t, x, r, p, X, \varphi(\cdot)) \\
& =\sup _{\alpha \in \mathcal{A}}\left\{\frac{1}{2} \operatorname{tr}\left[a^{\alpha}(t, x) X\right]+b^{\alpha}(t, x) \cdot p+\mathcal{I}^{\alpha} \varphi-c^{\alpha}(t, x) r+f^{\alpha}(t, x)\right\},
\end{aligned}
$$

where the integral operator $\mathcal{I}^{\alpha}$ is defined as

$$
\begin{aligned}
& \mathcal{I}^{\alpha} \varphi(t, x) \\
& =\int_{E}\left[\varphi\left(t, x+\eta^{\alpha}(x, z)\right)-\varphi(t, x)-\mathbf{1}_{|z|<1} \eta^{\alpha}(x, z) \cdot D \varphi(t, x)\right] \nu(d z),
\end{aligned}
$$

Date: March 5, 2008.

2000 Mathematics Subject Classification. 45K05, 49L25, 65M12, 65L70.

Key words and phrases. Integro-partial differential equation, viscosity solution, finite difference scheme, error estimate, stochastic optimal control, Lévy process, Bellman equation.

This research is supported by the Research Council of Norway through an Outstanding Young Investigators Award (KHK) and partially through the project "Integro-PDEs: Numerical methods, Analysis, and Applications to Finance". 
and $E=\mathbb{R}^{M} \backslash\{0\}$ ( $M$ integer) and $\nu(d z)$ is a positive Radon measure on $E$ - the so-called Lévy measure possessing at most a second order singularity at the origin and typically exponential decay at infinity.

The set $\mathcal{A}$, the value set of all admissible controls, is a compact metric space and the coefficients $a^{\alpha}, \eta^{\alpha}, b^{\alpha}, c^{\alpha}, f^{\alpha}, u_{0}$ are sufficiently regular functions taking values in $\mathbb{R}^{d \times d}, \mathbb{R}^{d}, \mathbb{R}^{d}, \mathbb{R}, \mathbb{R}, \mathbb{R}$ respectively. In this paper we will need $F$ to have special structure. The precise structure and assumptions on the coefficients will be given in the next section.

Equation (1.1) is degenerate parabolic since we will allow (i) the diffusion matrices $a^{\alpha}(t, x)$ merely to be non-negative definite and (ii) the jump vector $\eta^{\alpha}(x, z)$ to be zero for some $\alpha, x, z$. In other words there is no (global) regularization in this problem, neither from the second derivative ("Laplacian smoothing") nor from the integral term ("fractional Laplacian smoothing"). In general equation (1.1) will therefore not have classical solutions. For the type nonlinearity and degeneracy present in (1.1) it is natural to interpret solutions in the viscosity sense. The viscosity solution theory for the second order nonlinear partial differential equations is now well developed and has become an essential tool to study the optimal control problems for pure diffusion processes. In the past few years, there has been a considerable effort to extend the theory of viscosity solution to the integro-PDEs 1, 2, 3, 8, 9, 10, 16, 17, 22. Although this theory is not as developed as its pure PDE counter part, it is good enough to provide existence, uniqueness, comparison principles, and some regularity results in certain situations.

Although this connection will not be exploited herein, equations of the form (1.1) appear as the Bellman equations associated to the optimal control of jump-diffusion processes (or Lévy processes) over a finite time horizon (see [22, 23]). Examples include various types of portfolio optimization problems in which the risky asset is driven by a Lévy process. The linear version of (1.1) is of particular relevance to pricing theory of European option. For more information on pricing theory and its relation to linear integro-partial differential equations, we refer to [12.

In this paper we focus on finite difference-quadrature type schemes for 1.1 and their convergence properties. To be more precise, we will derive error estimates for numerical schemes for non-local equations of the form (1.1). There is a considerable literature addressing the issue of convergence of approximate (numerical) solutions to second order PDEs in the viscosity solution framework, see for example 17, 13, 14. The question of error estimate for numerical schemes, including finite difference type, is much more difficult and remained open until the recent works by Krylov 21, 19, 20] and Barles \& Jakobsen [4, 5, 6, 15].

On the other hand, finding error estimate for approximation schemes for fully nonlinear integro-PDEs is largely an untouched area with very few published results. In a recent development [18; Jakobsen, Karlsen and La Chioma have given a general framework for proving error estimates in the stationary case. To apply this framework strong assumptions are placed on the schemes, and in 18 they are verified only when the diffusion matrices $a^{\alpha}$ is independent of the space variable $x$. In this paper and the complimentary paper [11] we essentially use the framework of 18 and show how to verify this assumption in (much) more difficult situations when $a^{\alpha}$ also depends on $x$. We emphasize that the general framework is presented in [18] and that this paper contains particular a application of that framework. 
In [11] we treat the stationary case and derive error estimates for a class of problems with $x$-depending diffusion matrices. However, the 'jump vector' $\eta^{\alpha}$ could not depend on $x$. In this paper, we treat (nonlocal) time-dependent problems allowing both the diffusion matrices and the jump terms $\eta^{\alpha}$ to depend on $x$, at least for a class of nonlinearities $F$. The main results are error estimates for finite difference-quadrature schemes which are compatible with the structure of $F$. Our work here extends the results and techniques of Krylov 21] to a nonlocal setting. It is these techniques that allow us to verify the assumptions prescribed by 18 .

Throughout the major part of this paper we assume that the Lévy measure $\nu(d z)$ sitting inside the integral operator (1.3) is bounded and compactly supported. In this case we can re-write the nonlinearity $F$ in 1.1 , possibly at the expense of changing $b^{\alpha}$, as follows

$$
\begin{aligned}
& \bar{F}(t, x, r, p, X, \varphi(\cdot)) \\
& =\sup _{\alpha \in \mathcal{A}}\left\{\frac{1}{2} \operatorname{tr}\left[a^{\alpha}(t, x) X\right]+b^{\alpha}(t, x) \cdot p+\mathcal{J}^{\alpha} \varphi-c^{\alpha}(t, x) r+f^{\alpha}(t, x)\right\}
\end{aligned}
$$

where the integral operator $\mathcal{J}^{\alpha}$ is defined as

$$
\mathcal{J}^{\alpha}(\varphi)(t, x)=\int_{E}\left[\varphi\left(t, x+\eta^{\alpha}(x, z)\right)-\varphi(t, x)\right] \nu(d z) .
$$

Then (1.1) takes the form

$$
u_{t}+\bar{F}\left(t, x, u, D u, D^{2} u, u(t, \cdot)\right)=0 \text { in } Q_{T} .
$$

The general case where the Lévy measure can be unbounded and has unbounded support, can always be reduced to this case by suitable (standard) truncations. To be more precise, we replace in equation (1.1) the domain $E$ and Lévy measure $\nu$ by a truncated domain $\{z: r<|z|<R\}$ and a truncated Lévy measure

$$
\nu_{r, R}(d z)=\mathbf{1}_{r<|z|<R} \nu(d z) .
$$

Then we solve this new equation numerically using the finite-difference-quadrature method proposed in this paper. The truncation error can be controlled, and the details of this truncation procedure and its error bound can be found in [18. In the last section of this paper we will provide a short description on the rate where the Lévy measure is singular and the cut-off is chosen optimally. Here we also give some results for the problem without truncation, but only in the case when $\eta$ does not depend on $x$.

The rest of this paper is organized as follows: Section 2 collects preliminary material, including basic notations, precise form of the equations along standing assumptions on the involved coefficients, and some well-posedness and regularity results for these equations. In Section 3 we present the approximation scheme, give existence, uniqueness, comparison, and regularity results, and state our main result. Section 4 consists of the detailed proofs of the results stated in Section 3. In Section 5 we address briefly the case of unbounded Lévy measures.

\section{Preliminaries}

We denote the set of all $d \times d$ symmetric matrices $X=\left(X_{i j}\right), i, j=1,2, \ldots, d$, by $\mathbb{S}^{d}$, and let $\mathbb{R}^{d}$ be the $d$-dimensional Euclidean space where points are denoted 
by points $x=\left(x^{1}, x^{2}, \ldots, x^{d}\right)$. For any $l \in \mathbb{R}^{d}$, we define the directional derivatives $D_{l}$ and $D_{l}^{2}$ as follows

$$
D_{l} u=u_{x^{i}} l^{i} \text { and } D_{l}^{2} u=u_{x^{i} x^{j}} l^{i} l^{j}
$$

where $i$ and $j$ runs from 1 to $d$ and the summation convention applies. In this paper $D_{t}$ will denote the time derivative while $D$ will denote the spatial gradient. We denote the various constants by $N$ or $N(\cdots)$ with or without subscripts. In the second case $N$ only depends on the quantities in the parenthesis. Let

$$
a_{ \pm}=a^{ \pm}=\frac{1}{2}(|a| \pm a) \text {. }
$$

For some set $U$, let $C_{b}(U), C^{2}(U)$ and $C^{1,2}\left(Q_{T}\right)$ denote the spaces of all functions that are bounded continuous, twice continuous differentiable, and continuous differentiable once in $t$ and twice in $x$ respectively. For a measurable function $u$ defined on $U$ we define the norm

$$
|u|_{0}=\operatorname{ess}_{\sup } x \in U|u(x)| .
$$

For bounded functions $u(t, x)$ and $v(x)$ which are Lipschitz continuous in $x$ and Hölder continuous with exponent $\frac{1}{2}$ in $t$, we also define

$$
\begin{gathered}
|u|_{1, \frac{1}{2}}=|u|_{0}+\sup _{\substack{x \neq y, t \neq s \\
x, y \in \mathbb{R}^{d} ; t, s \in \mathbb{R}}} \frac{|u(x, t)-u(y, s)|}{|x-y|+|t-s|^{\frac{1}{2}}}, \\
|v|_{1}=|v|_{0}+\sup _{\substack{x \neq y, x, y \in \mathbb{R}^{n}\\
}} \frac{|v(x)-v(y)|}{|x-y|} .
\end{gathered}
$$

The Integro-PDE (1.5) we consider in this paper takes the following form:

$$
u_{t}+\sup _{\alpha \in \mathcal{A}}\left\{\mathcal{L}^{\alpha} u(t, x)+f^{\alpha}(t, x)+\mathcal{J}^{\alpha} u(t, x)\right\}=0,
$$

with the terminal condition $(1.2)$. Here $\mathcal{L}^{\alpha}$ is defined as

$$
\mathcal{L}^{\alpha} u:=a_{k}^{\alpha} D_{l_{k}}^{2} u+b_{k}^{\alpha} D_{l_{k}} u-c^{\alpha} u ; a_{k}^{\alpha}:=\frac{1}{2}\left(\sigma_{k}^{\alpha}\right)^{2},
$$

for $k= \pm 1, \pm 2, \ldots, \pm d_{1}, \mathcal{J}^{\alpha}$ is defined in (1.4), and

$$
a^{\alpha}(t, x)=\frac{1}{2} \sigma^{\alpha}\left(\sigma^{\alpha}\right)^{T} \quad \text { where } \quad \sigma_{i k}^{\alpha}(t, x)=l_{k}^{i} \sigma_{k}^{\alpha}(t, x) .
$$

Furthermore, $l_{k} \in \mathbb{R}^{d}, \sigma_{k}^{\alpha}(t, x), b_{k}^{\alpha}(t, x), c^{\alpha}(t, x)$ are real valued functions, and $\eta^{\alpha}(x, z)$ is an $\mathbb{R}^{d}$-valued function.

We will assume that there are constants $K>1$ and $\lambda \geq 0$ such that the following assumptions are satisfied:

(A.1) $\sigma_{k}^{\alpha}(t, x), \eta^{\alpha}(x, z), b_{k}^{\alpha}(t, x), c^{\alpha}(t, x), f^{\alpha}(t, x), u_{0}(x)$ are continuous in $t, x, z, \alpha$, and satisfy

$$
l_{k}=-l_{-k}, \quad \sigma_{k}^{\alpha}=\sigma_{-k}^{\alpha}, \quad b_{k}^{\alpha} \geq 0, \quad c^{\alpha} \geq \lambda, \quad\left|l_{k}\right| \leq K .
$$

(A.2) The measure $\nu$ is a positive Radon measure on $E$ satisfying

$$
\int_{E} \nu(d z)<\infty \quad \text { and } \quad \int_{E \backslash B(0, K)} \nu(d z)=0 .
$$

(A.3) For all $\alpha, z, k,\left|\sigma_{k}^{\alpha}\right|_{\frac{1}{2}, 1}+\left|b_{k}^{\alpha}\right|_{\frac{1}{2}, 1}+\left|c^{\alpha}\right|_{\frac{1}{2}, 1}+\left|f^{\alpha}\right|_{\frac{1}{2}, 1}+\left|\eta^{\alpha}(\cdot, \cdot)\right|_{1}+\left|u_{0}\right|_{1} \leq K$.

Next we define the concept of viscosity solutions for 2.1. 
Definition 2.1. $v \in U S C\left(Q_{T}\right)\left(v \in L S C\left(Q_{T}\right)\right)$ is a viscosity subsolution (supersolution) of (2.1) if for every $(t, x) \in Q_{T}$ and $\phi \in C^{1,2}\left(Q_{T}\right)$ such that $(t, x)$ is a global maximizer (global minimizer) for $v-\phi$,

$$
\phi_{t}+\sup _{\alpha \in \mathcal{A}}\left\{a_{k}^{\alpha} D_{l_{k}}^{2} \phi+b_{k}^{\alpha} D_{l_{k}} \phi-c^{\alpha} v(t, x)+f^{\alpha}(t, x)+\mathcal{J}^{\alpha} \phi(t, x)\right\} \geq 0(\leq 0) .
$$

We say that $v$ is a viscosity solution of 2.1) if $v$ is simultaneously a sub- and supersolution of 2.1.

Remark 2.1. The inequalities in definition (2.1) are reversed for the sub- and supersolution compared to the usual definition (see, e.g., [16]). A time change $t \rightarrow T-t$ will transform this terminal value problem into an (equivalent) initial value problem where the usual definition applies.

Remark 2.2. Contrary to the usual case [16, there are no restrictions on the growth of $\phi$ in this definition. The reason is that the integral term is well defined whatever the growth of $\phi$ is since the Lévy measure $\nu$ has compact support.

For a detailed treatment for the viscosity solutions of parabolic integro-partial differential equations we suggest [16] and references therein.

In order to get the final error estimate we will use a regularizing procedure introduced by Krylov, called the method of shaking the coefficients. This procedure requires the following auxiliary equation,

$$
\begin{aligned}
& u_{t}+\sup _{(\alpha, r, y) \in \mathcal{A} \times \Lambda \times B_{1}}\left[\mathcal{L}^{\alpha}\left(t+\epsilon^{2} r, x+\epsilon y\right) u+f^{\alpha}\left(t+\epsilon^{2} r, x+\epsilon y\right)\right. \\
& \left.+\int_{E}\left(u\left(t, x+\eta^{\alpha}(x+\epsilon y, z)\right)-u(t, x)\right) \nu(d z)\right]=0
\end{aligned}
$$

in $Q_{T}$ with the terminal data 1.2 where $\epsilon \in \mathbb{R}$ is a constant, $B_{1}=\left\{x \in \mathbb{R}^{d}\right.$ : $|x|<1\}$, and $\Lambda=(-1,0)$. We close this section by stating a well-posedness and continuous dependence result for 1.5 and $(2.2)$. A proof can be found in [16].

Theorem 2.1. Assume (A.1), (A.2), (A.3) hold, then there exist unique solutions $v$ and $v^{\epsilon}$ respectively to the terminal value problems $(2.1) /(1.2)$ and $(2.2) /(1.2)$ and a constant $N$ depending only on $d_{1}, K, T, \nu$ such that

$$
\left|v^{\epsilon}-v\right|_{0} \leq N|\epsilon| \text { and }\left|v^{\epsilon}\right|_{1, \frac{1}{2}}+|v|_{1, \frac{1}{2}} \leq N .
$$

Furthermore, a comparison principle holds: If $u$ and $\bar{u}$ are bounded sub- and supersolutions of either $2.1 / 1.2$ or $2.2 / 1.2$, then $u \leq \bar{u}$ in $\bar{Q}_{T}$.

\section{The DIFFERENCE-QUADRATURE SCHEME AND CONVERGENCE RATE}

We begin this section with a description of a finite difference approximation to (2.1). For $h_{1}, h_{2}, \tau>0, l \in \mathbb{R}^{d},(t, x) \in[0, T) \times \mathbb{R}^{d}$ we define the following finite difference operators:

$$
\begin{aligned}
\delta_{h_{1}, l} u(t, x) & =\frac{u\left(t, x+h_{1} l\right)-u(t, x)}{h_{1}}, \\
\Delta_{h_{1}, l} u(t, x) & =\frac{u\left(t, x+h_{1} l\right)-2 u(t, x)+u\left(t, x-h_{1} l\right)}{h_{1}^{2}}, \\
\delta_{\tau} u(t, x) & =\frac{u(t+\tau, x)-u(t, x)}{\tau},
\end{aligned}
$$




$$
\delta_{\tau}^{T} u(t, x)=\frac{u\left(t+\tau_{T}(t), x\right)-u(t, x)}{\tau}, \tau_{T}(t)=(t+\tau) \wedge T .
$$

To discretize the integral in 2.1 we introduce a quadrature rule

$$
I_{h_{2}}(f)=\sum_{p \in h_{2} \mathbb{Z}^{M}} k_{p} f(p), \quad k_{p} \geq 0, \quad k_{p}=0 \text { for }|p|>K,
$$

where $p \in h_{2} \mathbb{Z}^{M}$ and $k_{p} \geq 0$ are the nodes and weights respectively. Since $k_{p} \geq 0$, this scheme is monotone. This assumption is crucial for the analysis and natural since the measure $\nu$ is positive. Note that the sum is finite since $k_{p}=0$ for $|p|>K$, and this is also natural since the measure $\nu$ has support in $|p| \leq K$. We also require the following consistency estimate (error estimate)

$$
\left|\int_{E} f \nu(d z)-I_{h_{2}}(f)\right| \leq \nu(E) L_{f} h_{2}
$$

for every Lipschitz function $f$ with Lipschitz constant $L_{f}$.

Remark 3.1. Many classical quadrature rules satisfy these assumptions, the simplest example being the Riemann sum approximation,

$$
I_{h_{2}}(f)=\sum_{p \in \subset h_{2} \mathbb{Z}^{m}} f(p) \nu\left(p+\left[0, h_{2}\right]^{M}\right) .
$$

Other examples include the Newton-Cotes quadratures of order less than 9. We refer to [18] for a more detailed discussion.

Now we are in a position to introduce the implicit difference-quadrature scheme:

$$
\delta_{\tau}^{T} u(t, x)+\sup _{\alpha \in \mathcal{A}}\left[\mathcal{L}_{h_{1}}^{\alpha}(t, x) u+f^{\alpha}(t, x)+\mathcal{J}_{h_{2}}^{\alpha} u\right]=0 \quad \text { in } \quad Q_{T},
$$

with the terminal condition 1.2 , where

$$
\begin{aligned}
\mathcal{L}_{h_{1}}^{\alpha} u & =a_{k}^{\alpha} \Delta_{h_{1}, l_{k}} u+b_{k}^{\alpha} \delta_{h_{1}, l_{k}} u-c^{\alpha} u, \\
\mathcal{J}_{h_{2}}^{\alpha} u & =I_{h_{2}}\left(u\left(t, x+\eta^{\alpha}(x, z)\right)-u(t, x)\right) .
\end{aligned}
$$

As a simple consequence of Taylors theorem, we have the following consistency bound (truncation error)

$$
\left|\mathcal{L}_{h_{1}}^{\alpha} g(x)-\mathcal{L}^{\alpha} g(x)\right| \leq N^{*}\left(h_{1}^{2} \sup _{y \in B_{K}(x)}\left|D_{y}^{4} g\right|+h_{1} \sup _{y \in B_{K}(x)}\left|D_{y}^{2} g\right|\right),
$$

for every four times differentiable function $g$ and $h_{1} \leq 1$, where $N^{*}$ is constant which only depends on $K, d_{1}$ and $B_{K}(x)=\{|x| \leq K\}$.

Remark 3.2. The solution $u$ of the approximation scheme $(3.3)$ is defined on $Q_{T}$, and not merely on a fixed grid. In part this is a technical trick to simplify the analysis, and the numerical solution defined on a grid should simply be the restriction of $u$ to the $h_{1}$-grid. Indeed, in the local PDE context the numerical scheme would be well-defined for functions defined only on the $h_{1}$-grid. However, due to the choice of numerical quadrature, this is not the case in our nonlocal setting, and the present scheme cannot be implemented on a computer as it stands. Nevertheless, this can be remedied easily by replacing the integrand by a suitable interpolant over the $h_{1}$ grid. If piecewise linear interpolation is used, monotonicity of the scheme is preserved and all the estimates obtained in this paper would still hold. From a mathematical point of view, the essential difficulties are already 
present in the scheme (3.3), so to avoid increasing the length of this paper we will defer the analysis of the scheme with "interpolation" to future work.

Remark 3.3. The effect of using the difference operator $\delta_{\tau}^{T}$ in $(3.3)$ is "piecewise constant interpolation in time of the solutions". It is equivalent to using the scheme with the operator $\delta_{\tau}$ and constant-in-time initial data on the strip $[-\tau, 0] \times \mathbb{R}^{d}$.

We have the following lemma ensuring the existence of unique solution for the finite difference equation $(3.3)$.

Lemma 3.1. Assume (A.1), (A.2), (A.3), and (3.1) hold. Then there is a unique bounded function $u(t, x)$ solving $(3.3) /(1.2)$.

Proof. For each time-level $t$, existence of such a solution can be proven if one know that such a solution exits for $t+\tau_{T}$ by the contraction mapping argument used in the stationary case in Lemma 3.1 in [11. Iterations, starting from terminal time $T$ then complete the proof.

Remark 3.4. It follows from the proof that the function $u(t, x)$ is continuous in $x$ but in general it will be discontinuous in $t$. However, $u$ will satisfy a discrete Hölder bound in $t$ (Theorem 3.4), so the size of discontinuities decrease to 0 as $\tau \rightarrow 0$.

For fixed $\tau>0$, define

$$
\overline{\mathcal{M}}_{T}=\{n \tau \wedge T: n=0,1,2,3 \ldots \ldots\} \times \mathbb{R}^{d}
$$

and $\mathcal{M}_{T}=\overline{\mathcal{M}}_{T} \cap[0, T) \times \mathbb{R}^{d}$. The scheme is well-defined on $\mathcal{M}_{T}$, and often we will deduce properties of the scheme on $\overline{\mathcal{M}}_{T}$ and subsequently translate them to the whole space $Q_{T}=\mathcal{M}_{T}+[0, \tau] \times\{0\}$. We have the following lemma whose proof is postponed to the next section.

Lemma 3.2. Assume (A.1), (A.2), (A.3), (3.1), (3.2) and $h_{1}<1$. Let $C$ be a constant and $u_{1}, u_{2}$ functions defined on $\mathcal{M}_{T}$, continuous in $x$ for each $t$, and for some constant $\mu>0$,

$$
\sup _{\mathcal{M}_{T}}\left|u_{i}(t, x) e^{-\mu|x|}\right|<\infty, \quad i=1,2 .
$$

If $u_{1}(T, x) \leq u_{2}(T, x)$ and

$$
\begin{array}{r}
\delta_{\tau}^{T} u_{1}+\sup _{\alpha \in \mathcal{A}}\left[\mathcal{L}_{h_{1}}^{\alpha} u_{1}+f^{\alpha}(t, x)+\mathcal{J}_{h_{2}}^{\alpha} u_{1}\right]+C \\
\geq \delta_{\tau}^{T} u_{2}+\sup _{\alpha \in A}\left[\mathcal{L}_{h_{1}}^{\alpha} u_{2}+f^{\alpha}(t, x)+\mathcal{J}_{h_{2}}^{\alpha} u_{2}\right],
\end{array}
$$

then there exists a constant $\tau^{*}>0$ depending only on $K, d_{1}, \mu, \nu(E)$ such that if $\tau \in\left(0, \tau^{*}\right)$,

$$
u_{1} \leq u_{2}+(T+\tau) C_{+} \text {in } \quad \overline{\mathcal{M}}_{T} .
$$

Furthermore, $\tau^{*}\left(K, d_{1}, \mu, \nu(E)\right) \rightarrow \infty$ as $\mu \downarrow 0$, and if $u_{1}, u_{2}$ are bounded, 3.6. holds for all $\tau>0$.

Corollary 3.3. Assume (A.1), (A.2), (A.3), (3.1), (3.2) and $h_{1}<1$. Then the solution $v_{\tau, h}$ of (3.3)-(1.2) satisfies

$$
\left|v_{\tau, h}\right|_{0} \leq K(T+\tau)+\left|u_{0}\right|_{0} .
$$

Proof. The function $\pm\left[K(T-t)+\left|u_{0}\right|_{0}\right]$ is supersolution/subsolution of (3.3)-(1.2) (remember $c^{\alpha} \geq \lambda \geq 0$ ), so the result follows from Lemma 3.4 . 
Consider the terminal value problem

$$
\begin{aligned}
& \delta_{\tau}^{T} u+\sup _{(\alpha, r, y) \in A \times \Lambda \times B_{1}}\left[\mathcal{L}_{h_{1}}^{\alpha}\left(t+\epsilon^{2} r, x+\epsilon y\right) u(t, x)\right. \\
& \left.3.7)+f^{\alpha}\left(t+\epsilon^{2} r, x+\epsilon y\right)+\sum_{p} k_{p}\left(u\left(t, x+\eta^{\alpha}(x+\epsilon y, p)\right)-u(t, x)\right)\right]=0 \text { in } Q_{T},
\end{aligned}
$$

with terminal data 1.2 . This is the difference-quadrature scheme corresponding to (2.2). By Lemma 3.1 and Corollary 3.3 there exists a unique bounded solution $v_{\tau, h}^{\epsilon}$ of this problem.

We have the following theorem, whos proof will be given in the next section.

Theorem 3.4. Assume (A.1), (A.2), (A.3), (3.1), (3.2), $0 \leq h_{1} \leq 1$, and $0 \leq$ $\tau \leq \tau_{0}$. If $\tau_{0}$ is small enough, there exists a constant $N$ (depending only on $\tau_{0}, \lambda, T, K, d_{1}$, and $\left.\nu(E)\right)$; such that for all $\epsilon \in \mathbb{R}$

$$
\begin{aligned}
\left|v_{\tau, h}^{\epsilon}(t, x)-v_{\tau, h}(t, x)\right| & \leq N|\epsilon|, \\
\left|v_{\tau, h}^{\epsilon}(t, x)-v_{\tau, h}^{\epsilon}(t, y)\right|+\left|v_{\tau, h}(t, x)-v_{\tau, h}(t, y)\right| & \leq N|x-y|, \\
\left|v_{\tau, h}^{\epsilon}(t, x)-v_{\tau, h}^{\epsilon}(s, x)\right|+\left|v_{\tau, h}(t, x)-v_{\tau, h}(s, x)\right| & \leq N\left(|t-s|^{\frac{1}{2}}+\tau^{\frac{1}{2}}\right),
\end{aligned}
$$

for all $(t, x),(s, y) \in \bar{Q}_{T}$.

Now, with the help of the results stated above, we are in a position to prove the main contribution of this paper, namely

Theorem 3.5. Assume (A.1), (A.2), (A.3), (3.1), (3.2), $0<h_{1}, h_{2}$, and $0<\tau \leq$ $\tau_{0}$. If $\tau_{0}, h_{1}, h_{2}$ are small enough, there exists a constant $N_{1}$ (depending only on $\left.\tau_{0}, \lambda, d_{1}, T, K, \nu(E)\right)$, such that

$$
\left|v-v_{\tau, h}\right|_{0} \leq N_{1}\left[\tau^{\frac{1}{4}}+h_{1}^{\frac{1}{2}}+h_{2}\right] .
$$

Proof. Take $\epsilon=\left(\tau+h_{1}^{2}+h_{2}^{4}\right)^{\frac{1}{4}}$ and let $\tau_{0}, h_{1}, h_{2}$ be sufficiently small such that $\varepsilon<1$. If $T<2 \epsilon^{2}$ then the theorem holds because by 3.10 and 2.3 and the definition of $\varepsilon$,

$$
\begin{aligned}
\sup _{\bar{Q}_{T}}\left|v_{\tau, h}-v\right| & \leq \sup _{\bar{Q}_{T}}\left|v_{\tau, h}-u_{0}\right|+\sup _{\bar{Q}_{T}}\left|u_{0}-v\right| \\
& \leq N\left(T^{\frac{1}{2}}+\tau^{\frac{1}{2}}\right) \leq N\left(\tau+h_{1}^{2}+h_{2}^{4}\right)^{\frac{1}{4}} .
\end{aligned}
$$

Next we consider the case $T>2 \epsilon^{2}$. First we prove the upper bound

$$
v-v_{\tau, h} \leq N\left(\tau^{\frac{1}{4}}+h_{1}^{\frac{1}{2}}+h_{2}\right) .
$$

For each $\alpha \in \mathcal{A}, r \in(-1,0)$ and $|y|<1$, equation (3.7) implies

$$
\begin{aligned}
& \delta_{\tau}^{T} v_{\tau, h}^{\epsilon}\left(t-\epsilon^{2} r, x-\epsilon y\right)+\mathcal{L}_{h_{1}}^{\alpha}(t, x) v_{\tau, h}\left(t-\epsilon^{2} r, x-\epsilon y\right)+f^{\alpha}(t, x) \\
& +\sum_{p} k_{p}\left[v_{\tau, h}^{\epsilon}\left(t-\epsilon^{2} r, x+\eta^{\alpha}(x, p)-\epsilon y\right)-v_{\tau, h}^{\epsilon}\left(t-\epsilon^{2} r, x-\epsilon y\right)\right] \leq 0
\end{aligned}
$$

for $(t, x) \in \bar{Q}_{T-\epsilon^{2}}$.

Now use Krylov's technique i.e. multiply inequality (3.12) with a mollifier and convolve. Let $\zeta \in C_{0}^{\infty}\left(\mathbb{R}^{d+1}\right)$ be our mollifier, a positive function with unit integral and having support in $\Lambda \times B_{1}$. Also denote,

$$
u^{(\epsilon)}(t, x)=\epsilon^{(-d-2)} \int_{\mathbb{R}^{d+1}} u(t-s, x-y) \zeta\left(\frac{s}{\epsilon^{2}}, \frac{y}{\epsilon}\right) d s d y
$$


Then multiplying 3.12 by $\epsilon^{-d-2} \zeta\left(s / \varepsilon^{2}, y / \varepsilon\right)$ and integrating with respect to $(s, y)$ we obtain, for each $\alpha \in \mathcal{A}$, on $\bar{Q}_{T-2 \epsilon^{2}}$

$$
\delta_{\tau}^{T} v_{\tau, h}^{\epsilon(\epsilon)}+\mathcal{L}_{h_{1}}^{\alpha}(t, x) v_{\tau, h}^{\epsilon(\epsilon)}+f^{\alpha}+\sum_{p} k_{p}\left(v_{\tau, h}^{\epsilon(\epsilon)}\left(t, x+\eta^{\alpha}(x, p)\right)-v_{\tau, h}^{\epsilon(\epsilon)}\right) \leq 0 .
$$

From 3.4, 3.2, and Taylor's formula we have

$$
\begin{aligned}
& \frac{\partial}{\partial t} v_{\tau, h}^{\epsilon(\epsilon)}+\mathcal{L}^{\alpha}(t, x) v_{\tau, h}^{\epsilon(\epsilon)}+f^{\alpha}+\int_{\mathbb{R}^{M} \backslash\{0\}}\left(v_{\tau, h}^{\epsilon(\epsilon)}\left(t, x+\eta^{\alpha}(x, z)\right)-v_{\tau, h}^{\epsilon(\epsilon)}\right) \nu(d z) \\
& \leq N\left(\tau\left|D_{t}^{2} v_{\tau, h}^{\epsilon(\epsilon)}\right|_{0, \bar{Q}_{T-2 \epsilon^{2}}}+h_{1}^{2}\left|D_{x}^{4} v_{\tau, h}^{\epsilon(\epsilon)}\right|_{0, \bar{Q}_{T-2 \epsilon^{2}}}+h_{1}\left|D_{x}^{2} v_{\tau, h}^{\epsilon(\epsilon)}\right|_{0, \bar{Q}_{T-2 \epsilon^{2}}}\right. \\
& \left.\quad+h_{2}\left|D_{x} v_{\tau, h}^{\epsilon(\epsilon)}\right|_{0, \bar{Q}_{T-2 \epsilon^{2}}}\right):=I \quad \text { in } \bar{Q}_{T-2 \epsilon^{2}} .
\end{aligned}
$$

Clearly $v_{\tau, h}^{\epsilon(\epsilon)}+\left(T-2 \epsilon^{2}-t\right) I$ is a classical supersolution to the equation (2.1) and hence a viscosity supersolution as well in $Q_{T-2 \epsilon^{2}}$. Now using the comparison principle (Theorem 2.1) we have

$$
v \leq v_{\tau, h}^{\epsilon(\epsilon)}+\left(T-2 \epsilon^{2}-t\right) I+\sup _{\left\{\left(T-2 \epsilon^{2}\right) \times \mathbb{R}^{d}\right\}}\left|v-v^{\epsilon(\epsilon)}\right| .
$$

Using properties of convolutions and regularity of $v_{\tau, h}^{\epsilon}$ (Theorem 3.4),

$$
\left|v_{\tau, h}^{\epsilon(\epsilon)}-v_{\tau, h}^{\epsilon}\right| \leq N \epsilon \quad \text { and } \quad \epsilon^{2 n-1}\left|D_{t}^{n} v_{\tau, h}^{\epsilon(\epsilon)}\right|_{0, \bar{Q}_{T-2 \epsilon^{2}}}+\epsilon^{n-1}\left|D_{x}^{n} v_{\tau, h}^{\epsilon(\epsilon)}\right|_{0, \bar{Q}_{T-2 \epsilon^{2}}} \leq N
$$

By the same reasoning as in the beginning of the proof, we also find

$$
\left|v\left(T-2 \epsilon^{2}, x\right)-v^{\epsilon(\epsilon)}\left(T-2 \epsilon^{2}, x\right)\right| \leq N \epsilon .
$$

By the above estimates, Theorem 3.4 and recalling that $\varepsilon^{4}=\tau+h_{1}^{2}+h_{2}^{4}$, we obtain

$$
\begin{aligned}
v & \leq v_{\tau, h}^{\epsilon}+N\left(\epsilon+\frac{\tau+h_{1}^{2}}{\epsilon^{3}}+\frac{h_{1}}{\epsilon}+h_{2}\right) \\
& \leq v_{\tau, h}+N\left(\epsilon+\frac{\tau+h_{1}^{2}}{\epsilon^{3}}+\frac{h_{1}}{\epsilon}+h_{2}\right) \\
& \leq v_{\tau, h}+N\left(\tau+h_{1}^{2}+h_{2}^{4}\right)^{\frac{1}{4}} \quad \text { in } \bar{Q}_{T-2 \epsilon^{2}} .
\end{aligned}
$$

By the regularity of $v, v_{\tau, h}$ and the argument given in the case $T<2 \varepsilon^{2}$, this estimate in fact holds in all of $\bar{Q}_{T}$. Moreover, it can be checked that all constants $N$ only depend on $\tau_{0}, \lambda, \nu(E), d_{1}, d, K$ and $T$. This completes the proof of 3.11.

The lower bound

$$
v_{\tau, h}-v \leq N\left(\tau^{\frac{1}{4}}+h_{1}^{\frac{1}{2}}+h_{2}\right)
$$

can be proved in a similar way. Interchange the role of the finite difference scheme and the equation 2.1 in the argument leading to 3.11 . Now it can be shown that $v^{\epsilon(\epsilon)}$ is a classical supersolution of 2.1 in $Q_{T-\epsilon^{2}}$. We skip the arguments since they are similar to the arguments for stationary integro-PDEs given in [18], see also [20, 15] for time-dependent pure PDEs.

By consistency 3.2 and $(3.4)$, regularity of $v^{\epsilon(\epsilon)}$, and properties of mollifiers, it follows that

$$
\delta_{\tau}^{T} v^{\epsilon(\epsilon)}+\sup _{\alpha \in A}\left[L_{h_{1}}^{\alpha} v^{\epsilon(\epsilon)}+f^{\alpha}+\mathcal{J}_{h_{2}}^{\alpha} v^{\epsilon(\epsilon)}\right] \leq N\left(\frac{\tau+h_{1}^{2}}{\epsilon^{3}}+\frac{h_{1}}{\epsilon}+h_{2}\right)
$$


in $\bar{Q}_{T-\epsilon^{2}-\tau}$, and the comparison result (Lemma 3.2 then gives

$$
v_{\tau, h} \leq v^{\epsilon(\epsilon)}+\sup _{Q_{T} \backslash Q_{T-\epsilon^{2}-\tau}}\left(v_{\tau, h}-v^{\epsilon(\epsilon)}\right)_{+}+N\left(\frac{\tau+h_{1}^{2}}{\epsilon^{3}}+\frac{h_{1}}{\epsilon}+h_{2}\right) .
$$

Since $\tau \leq \varepsilon^{4} \leq \varepsilon^{2} \leq 1$ and hence $Q_{T} \backslash Q_{T-\epsilon^{2}-\tau} \subset Q_{T} \backslash Q_{T-2 \epsilon^{2}}$,

$$
\sup _{Q_{T} \backslash Q_{T-\epsilon^{2}-\tau}}\left(v_{\tau, h}-v^{\epsilon(\epsilon)}\right)_{+} \leq \sup _{Q_{T} \backslash Q_{T-2 \epsilon^{2}}}\left|v_{\tau, h}-v^{\epsilon(\epsilon)}\right| \leq N \epsilon,
$$

where the last inequality was proved at the start of this proof. This estimate, (3.15), and the definition of $\varepsilon$, implies the lower bound (3.14). It can be checked that $N$ depends only on $\nu(E), K, d_{1}, d$ and $T$.

\section{Proofs of the Results stated in Section 3}

In this section we prove comparison and Lipschitz continuity results for the solution of the difference-quadrature scheme (3.3), 1.2. As an application of the Lipschitz result we derive a continuous dependence estimate for the scheme. Although the basic ideas behind our proofs come from Krylov 21, the nonlocal nature of the problem adds to some extra difficulties and they do not allow us to adopt the "local" approach of Krylov. Our approach is more direct and we employ some new techniques.

We begin by stating some auxiliary results. To this end, we need the translation operator

$$
T_{h_{1}, l} u(x):=u\left(x+h_{1} l\right) .
$$

We now give some technical lemmas whose proofs can be found in [21].

Lemma 4.1. For any functions $u(x), v(x), h_{1}>0$. and $l \in \mathbb{R}^{d}$ we have

$$
\begin{aligned}
T_{h_{1},-l} T_{h_{1}, l} u= & u, \\
T_{h_{1}, l} \delta_{h_{1},-l}= & \delta_{h_{1},-l} T_{h_{1}, l}=-T_{h_{1},-l} \delta_{h_{1}, l}=-\delta_{h_{1}, l} T_{h_{1},-l}=-\delta_{h_{1},-l}, \\
\delta_{h_{1}, l}(u v)= & v \delta_{h_{1}, l}(u)+T_{h_{1}, l} u \delta_{h_{1}, l}(v), \\
= & u \delta_{h_{1}, l}(v)+v \delta_{h_{1}, l}(u)+h_{1}\left(\delta_{h_{1}, l}(v)\right)\left(\delta_{h_{1}, l}(u)\right) \\
\Delta_{h_{1}, l}(u v)= & u \Delta_{h_{1}, l}(v)+v \Delta_{h_{1}, l}(u)+\left(\delta_{h_{1}, l}(v)\right)\left(\delta_{h_{1}, l}(u)\right) \\
& \quad+\left(\delta_{h_{1},-l}(v)\right)\left(\delta_{h_{1},-l}(u)\right) .
\end{aligned}
$$

In particular,

$$
\Delta_{h_{1}, l}\left(u^{2}\right)=2 u \Delta_{h_{1}, l} u+\left(\delta_{h_{1}, l} u\right)^{2}+\left(\delta_{h_{1},-l} u\right)^{2} .
$$

Lemma 4.2. Let $u, v, w$ be functions on $\mathbb{R}^{d}, l, x_{0} \in \mathbb{R}^{d}, h_{1}>0$. Assume that $v\left(x_{0}\right) \leq 0$. Then at $x_{0}$ it holds

$$
\begin{array}{r}
-\delta_{h_{1}, l} v \leq \delta_{h_{1}, l}\left(v_{-}\right),-\Delta_{h_{1}, l} v \leq \Delta_{h_{1}, l}\left(v_{-}\right), \\
\left|\Delta_{h_{1}, l} u\right| \leq\left|\delta_{h_{1},-l}\left(\left(\delta_{h_{1}, l} u\right)_{-}\right)\right|+\left|\delta_{h_{1}, l}\left(\left(\delta_{h_{1},-l} u\right)_{-}\right)\right|, \\
\left|\Delta_{h_{1}, l} u\right| \leq\left|\delta_{h_{1},-l}\left(\left(\delta_{h_{1}, l} u\right)_{+}\right)\right|+\left|\delta_{h_{1}, l}\left(\left(\delta_{h_{1},-l} u\right)_{+}\right)\right| .
\end{array}
$$

Now we prove Lemma 3.2 .

Proof of Lemma 3.2: Let $T^{\prime}$ be the smallest $j \tau$ which exceeds $T$, where $j \in \mathbb{N}$. A solution to the equation $(3.3)$ on $\overline{\mathcal{M}}_{T}$ could be viewed as a solution to the same on $\overline{\mathcal{M}}_{T^{\prime}}$ after trivially redefining the function on $\left\{T^{\prime}\right\} \times \mathbb{R}^{d}$. So without loss of generality we assume that $T=T^{\prime}$. 
From 3.5 we then have in $\mathcal{M}_{T}$, for $u=u_{1}-u_{2}$

$$
\delta_{\tau} u+\sup _{\alpha \in \mathcal{A}}\left[\mathcal{L}_{h_{1}}^{\alpha} u+\sum_{p \in h_{2} \mathbb{Z}^{M}} k_{p}\left(u\left(t, x+\eta^{\alpha}(x, p)\right)-u(t, x)\right)\right]+C \geq 0 .
$$

Let $w=u-C_{+}(T-t)$ and note that

$$
\begin{aligned}
& \delta_{\tau} w+\sup _{\alpha \in \mathcal{A}}\left[\mathcal{L}_{h_{1}}^{\alpha} w+\sum_{p \in h_{2} \mathbb{Z}^{M}} k_{p}\left(w\left(t, x+\eta^{\alpha}(x, p)\right)-w(t, x)\right)\right] \\
& \geq \delta_{\tau} u+\sup _{\alpha \in \mathcal{A}}\left[\mathcal{L}_{h_{1}}^{\alpha} u+\sum_{p \in h_{2} \mathbb{Z}^{M}} k_{p}\left(u\left(t, x+\eta^{\alpha}(x, p)\right)-u(t, x)\right)\right]+C_{+}+\lambda C_{+}(T-t) \\
& \geq 0,
\end{aligned}
$$

and hence for $\varepsilon>0$,

$$
w+\epsilon \delta_{\tau} w+\epsilon \sup _{\alpha \in A}\left[\mathcal{L}_{h_{1}}^{\alpha} w+\sum_{p \in h_{2} \mathbb{Z}^{M}} k_{p}\left(w\left(t, x+\eta^{\alpha}(x, p)\right)-w(t, x)\right)\right] \geq w .
$$

For any $\psi \geq w$, we can choose $\epsilon$ small enough so that in $\mathcal{M}_{T}$,

$$
\begin{aligned}
& \psi+\epsilon \delta_{\tau} \psi+\epsilon \sup _{\alpha \in A}\left[\mathcal{L}_{h_{1}}^{\alpha} \psi+\sum_{p \in h_{2} \mathbb{Z}^{M}} k_{p}\left(\psi\left(t, x+\eta^{\alpha}(x, p)\right)-\psi(t, x)\right)\right] \\
& \geq w+\epsilon \delta_{\tau} w+\epsilon \sup _{\alpha \in A}\left[\mathcal{L}_{h_{1}}^{\alpha} w+\sum_{p \in h_{2} \mathbb{Z}^{M}} k_{p}\left(w\left(t, x+\eta^{\alpha}(x, p)\right)-w(t, x)\right)\right] \geq w .
\end{aligned}
$$

For a constant $\gamma$ and $\langle x\rangle:=\sqrt{1+x^{2}}$, we define the functions $\xi(t), \beta(x)$, and $\zeta(t, x)$ on $\overline{\mathcal{M}}_{T}$ in the following way:

$$
\begin{aligned}
& \xi(T)=1, \xi(t)=\gamma^{-1} \xi\left(t+\tau_{T}(t)\right) \text { if } t \in[0, T) \\
& \beta(x)=\cosh (\mu\langle x\rangle), \zeta(t, x)=\xi(t) \beta(x) .
\end{aligned}
$$

Note that $\xi$ is recursively defined. By straightforward computations we have,

$$
\begin{aligned}
& \sup _{\alpha \in \mathcal{A}}\left[\mathcal{L}_{h_{1}}^{\alpha} \beta+\sum_{p \in h_{2} \mathbb{Z}^{M}} k_{p}\left(\beta\left(x+\eta^{\alpha}(x, p)\right)-\beta(x)\right)\right] \\
& \leq \sup _{\alpha \in \mathcal{A}} \mathcal{L}^{\alpha} \beta+N_{1}\left(h_{1}^{2}+h_{1}\right) \cosh (\mu\langle x\rangle+K)+N_{2}(\nu(E), \mu) \cosh (\mu\langle x\rangle+K) \\
& \leq N_{2} \cosh (\mu\langle x\rangle+K) .
\end{aligned}
$$

Since $\delta_{\tau} \xi(t)=\frac{\gamma-1}{\tau} \xi(t)$ and $\cosh (\mu\langle x\rangle+K) \leq e^{K} \cosh (\mu\langle x\rangle)$, it follows that

$$
\begin{aligned}
& \delta_{\tau} \zeta+\sup _{\alpha \in A}\left[\mathcal{L}_{h_{1}}^{\alpha} \zeta+\sum_{p \in h_{2} \mathbb{Z}^{M}} k_{p}\left(\zeta\left(t, x+\eta^{\alpha}(x, p)\right)-\zeta(t, x)\right)\right] \\
& \leq \tau^{-1}(\gamma-1) \zeta+N_{3} \zeta=\kappa(\gamma) \zeta
\end{aligned}
$$

where $N_{3}=N_{2} e^{K}$ and $\kappa(\gamma)=\tau^{-1}(\gamma-1)+N_{3}$. We take $\tau *=N_{3}^{-1}$ and let $\tau<\tau^{*}$. Then $\kappa(0)<0$ and $\kappa(1) \geq 0$ and hence we can choose $\gamma$ so that $\kappa<0$ and $1+\kappa \epsilon>0$ for all $\varepsilon$ small enough.

Now set $N=\sup _{\overline{\mathcal{M}}_{T}} \frac{w_{+}}{\zeta}$. Taking $\psi=N \zeta$ and $\varepsilon$ small enough, 4.3 leads to

$$
\begin{aligned}
& N \zeta(1+\kappa \epsilon)=N \zeta+k \epsilon N \zeta \\
& \geq \psi+\epsilon \delta_{\tau}^{T} \psi+\epsilon \sup _{\alpha \in A}\left[\mathcal{L}_{h_{1}}^{\alpha} \psi+\sum_{p \in h_{2} \mathbb{Z}^{M}} k_{p}\left(\psi\left(t, x+\eta^{\alpha}(x, p)\right)-\psi(t, x)\right)\right] \geq w .
\end{aligned}
$$


in $\mathcal{M}_{T}$. But $w(T, x)$ is negative by definition, so the inequality holds on entire $\overline{\mathcal{M}}_{T}$. By the definition of $N$, we then have $N(1+\kappa \epsilon) \geq N$. Since $\kappa<0$, we conclude that $N=0$ and hence $w \leq 0$ and $(3.6)$ follows. The remaining part of the lemma becomes obvious if we choose $N_{1}=N_{2}=N_{3}=0$

Next we state and prove the key technical result of this paper.

Theorem 4.3. Assume (A.1), (A.3), (A.2), (3.1) and (3.2) hold. Let $u(t, x)$ be a function on $\overline{\mathcal{M}}_{T}$ solving (3.3) with $|u(T, \cdot)|_{1}<\infty$. There is a constant $N>0$, depending only on $K, d_{1}, d$ and the Lévy measure $\nu$, such that, if there is a number $c_{0} \geq 0$ satisfying

$$
\lambda+\frac{1-e^{-c_{0} \tau}}{\tau}>N
$$

then for every $0<\epsilon<K h_{1}, l \in \mathbb{R}^{d}$,

$$
\left|\delta_{\epsilon, \pm l} u(t, x)\right| \leq N_{1}(1 \vee|l|)\left(1+\sup _{k, x}\left|\delta_{h_{1}, l_{k}} u(T, \cdot)\right|+\sup _{x}\left|\delta_{\epsilon, \pm l} u(T, \cdot)\right|\right) \quad \text { in } \quad \overline{\mathcal{M}}_{T},
$$

where $N_{1}$ only depend on $T, \lambda, c_{0}, K, d, d_{1}, \nu(E)$.

Proof. We start by introducing a few additional notations. Let $r$ and $k$ be indices running through $\left\{ \pm 1, \pm 2 \ldots . . \pm\left(d_{1}+1\right)\right\}$ and $\left\{ \pm 1, \pm 2, \ldots . \pm d_{1}\right\}$ respectively, let $0<\varepsilon \leq K h_{1}$, and define

$$
h_{k}=h_{1}, k= \pm 1, \pm 2, \ldots . . \pm d_{1}, h_{ \pm\left(d_{1}+1\right)}=\epsilon, l_{ \pm\left(d_{1}+1\right)}= \pm l .
$$

Choose a constant $c_{0} \geq 0$, let $T^{\prime}$ be the least $n \tau, n=1,2,3, \ldots$, such that $n \tau \geq T$, and define

$$
\begin{aligned}
& \xi(t)=e^{c_{0} t} \quad \text { if } \quad t<T^{\prime}, \xi(T)=e^{c_{o} T^{\prime}} \text { otherwise; } \\
& v=\xi u ; \\
& v_{r}=\delta_{h_{r}, l_{r}} v \quad \text { if } \quad r= \pm 1, \pm 2, \ldots \ldots \pm d_{1} \\
& v_{ \pm\left(d_{1}+1\right)}=\frac{v(t, x \pm \epsilon l)-v(t, x)}{\epsilon(1 \vee|l|)} \\
& M=\sup _{(t, x) \in \bar{Q}_{T}}|v(t, x)|, \quad M_{1}=\sup _{r, x, l, t}\left|v_{r}\right|
\end{aligned}
$$

Now define

$$
W(t, x, l)=\sum_{r}\left(v_{r}^{-}\right)^{2} \quad \text { and } \quad V(t, x, l)=W(t, x, l)-\delta C(x),
$$

where $\delta>0$ and $C(x) \in C^{2}\left(\mathbb{R}^{d}\right)$ is positive, convex, and satisfy

$$
\lim _{|x| \rightarrow \infty} C(x)=\infty \text { and }|D C|_{0}+\left|D^{2} C\right|_{0}<\infty .
$$

To prove the theorem we have to find a bound on $M_{1}$ which is independent of the discretization constants. We will derive such a bound for $W$, and towards the end of the proof we will show that this bound implies the sought after bound on $M_{1}$.

From the properties of $C(x)$, it is clear that $V(t, x, l)$ is bounded above and that there exists a point $\left(t_{0}, x_{0}, l_{0}\right) \in \overline{\mathcal{M}}_{T} \times \mathbb{R}^{d}$ such that

$$
V\left(t_{0}, x_{0}, l_{0}\right)=\sup _{(t, x, l)} V(t, x, l) .
$$


If $t_{0}=T$, then

$$
V(T, \cdot) \leq W(T, \cdot) \leq N\left(d_{1}\right) e^{2 c_{0} T^{\prime}}\left(\sup _{r, x} u_{r}(T, x)\right)^{2},
$$

and the theorem is true by Lipschitz continuity of $u(T, x)$.

From now on we take $t_{0}<T$. By the definition of supremum, there is a sequence of control parameters $\left(\alpha_{n}\right) \in \mathcal{A}$ (depending on the maximum point $\left(x_{0}, t_{0}, l_{0}\right)$ ) such that

$$
\begin{aligned}
& \lim _{n \rightarrow \infty}\left[\mathcal{L}_{h_{1}}^{\alpha_{n}}\left(t_{0}, x_{0}\right) u\left(t_{0}, x_{0}\right)+f^{\alpha_{n}}\left(t_{0}, x_{0}\right)+\mathcal{J}_{h_{2}}^{\alpha_{n}} u\left(t_{0}, x_{0}\right)\right] \\
& =\sup _{\alpha \in \mathcal{A}}\left[\mathcal{L}_{h_{1}}^{\alpha}\left(t_{0}, x_{0}\right) u\left(t_{0}, x_{0}\right)+f^{\alpha}\left(t_{0}, x_{0}\right)+\mathcal{J}_{h_{2}}^{\alpha}\left(u\left(t_{0}, x_{0}\right)\right)\right] .
\end{aligned}
$$

By assumption (A.3) and the Arzela-Ascoli theorem there is a subsequence $\left\{\alpha_{n}\right\}$ and functions $\bar{a}_{k}, b_{k}, \bar{c}, \bar{f}, \bar{\eta}$, such that

$$
\left(a_{k}^{\alpha_{n}}, b_{k}^{\alpha_{n}}, c^{\alpha_{n}}, f^{\alpha_{n}}, \eta^{\alpha_{n}}\right) \rightarrow\left(\bar{a}_{k}, \bar{b}_{k}, \bar{c}, \bar{f}, \bar{\eta}\right) \quad \text { locally uniformly. }
$$

Obviously, $\bar{a}_{k}, \bar{b}_{k}, \bar{c}, \bar{f}, \bar{\eta}$ also satisfy (A.1) and (A.3). Moreover since $u$ solve (3.3),

$$
\begin{aligned}
& \delta_{\tau}^{T} u+\bar{a}_{k} \Delta_{h_{1}, l_{k}} u+\bar{b}_{k} \delta_{h_{1}, l_{k}} u-\bar{c} u+\bar{f} \\
& +\sum_{p \in h_{2} \mathbb{Z}^{M}} k_{p}\left(u\left(t_{0}, x_{0}+\bar{\eta}\left(x_{0}, p\right)\right)-u\left(t_{0}, x_{0}\right)\right)=0,
\end{aligned}
$$

at the point $\left(t_{0}, x_{0}\right)$, while at the points $\left(t_{0}, x_{0}+h_{r} l_{r}\right)$,

$$
\begin{aligned}
& \delta_{\tau}^{T} u+\bar{a}_{k} \Delta_{h_{1}, l_{k}} u+\bar{b}_{k} \delta_{h_{1}, l_{k}} u-\bar{c} u+\bar{f} \\
& +\left.\sum_{p \in h_{2} \mathbb{Z}^{M}} k_{p}(u(\cdot, \cdot+\bar{\eta}(\cdot, p))-u(\cdot, \cdot))\right|_{\left(t_{0}, x_{0}+h_{r} l_{r}\right)} \leq 0 .
\end{aligned}
$$

The last inequality holds at every point in $Q_{T}$. For simplicity we now drop the 0 subscript and rename the maximum point $(x, t, l)$. Replacing $u$ by $\xi^{-1} v$ in (4.6) and (4.7) we get

$$
\begin{aligned}
& \delta_{\tau}^{T}\left(\xi^{-1} v\right)+\xi^{-1}\left(\bar{a}_{k} \Delta_{h_{1}, l_{k}} v+\bar{b}_{k} \delta_{h_{1}, l_{k}} v-\bar{c} v+\bar{f}\right. \\
& \left.+\sum_{p \in h_{2} \mathbb{Z}^{M}} k_{p}(v(t, x+\bar{\eta}(x, p))-v(t, x))\right)=0
\end{aligned}
$$

at the point $(t, x)$ and for each $r$, and at the points $\left(t, x+h_{r} l_{r},\right)$ we have

$$
\begin{aligned}
& {\left[\delta_{\tau}^{T}\left(\xi^{-1} v\right)+\xi^{-1}\left(\bar{a}_{k} \Delta_{h_{1}, l_{k}} v+\bar{b}_{k} \delta_{h_{1}, l_{k}} v-\bar{c} v+\bar{f}\right.\right.} \\
& \left.\left.+\sum_{p \in h_{2} \mathbb{Z}^{M}} k_{p}(v(\cdot, \cdot+\bar{\eta}(\cdot, p))-v(\cdot, \cdot))\right)\right]\left.\right|_{\left(t, x+h_{r} l_{r}\right)} \leq 0 .
\end{aligned}
$$

Subtracting (4.8) from (4.9) and dividing the result by $h_{r}$, for $r= \pm 1, \pm 2, \ldots ., \pm d_{1}$, and by $\epsilon(|l| \vee 1)$ for $r= \pm\left(d_{1}+1\right)$, and using the product rule for difference quotients (Lemma 4.1) we get

$$
\delta_{\tau}^{T}\left(\xi^{-1} v_{r}\right)+\xi^{-1}\left[\bar{a}_{k} \Delta_{h_{k}, l_{k}} v_{r}+I_{1 r}+I_{2 r}+I_{3 r}+I_{4 r}+I_{5 r}\right] \leq 0,
$$

where there is no summation with respect to $r$. Here

$$
I_{1 r}= \begin{cases}\left(\delta_{h_{r}, l_{r}} \bar{a}_{k}\right) \Delta_{h_{k}, l_{k}} v, & \text { if } r \neq \pm\left(d_{1}+1\right) \\ \frac{1}{(1 \vee|l|)}\left(\delta_{h_{r}, l_{r}} \bar{a}_{k}\right) \Delta_{h_{k}, l_{k}} v, & \text { if } r= \pm\left(d_{1}+1\right),\end{cases}
$$




$$
\begin{aligned}
& I_{2 r}=h_{r}\left(\delta_{h_{r}, l_{r}} \bar{a}_{k}\right) \Delta_{h_{k}, l_{k}} v_{r}, \\
& I_{3 r}= \begin{cases}\left(T_{h_{r}, l_{r}} \bar{b}_{k}\right) \delta_{h_{k}, l_{k}} v_{r}+\left(\delta_{h_{r}, l_{r}} \bar{b}_{k}\right) \delta_{h_{k}, l_{k} v,} & \text { if } r \neq \pm\left(d_{1}+1\right) \\
\left(T_{h_{r}, l_{r}} \bar{b}_{k}\right) \delta_{h_{k}, l_{k}} v_{r}+\frac{1}{(1 \vee|l|)}\left(\delta_{h_{r}, l_{r}} \bar{b}_{k}\right) \delta_{h_{k}, l_{k}} v, & \text { if } r= \pm\left(d_{1}+1\right),\end{cases} \\
& I_{4 r}= \begin{cases}-\left(\delta_{h_{r}, l_{r}} \bar{c}\right) v-\left(T_{h_{r}, l_{r}} \bar{c}\right) v_{r}+\xi \delta_{h_{r}, l_{r}} \bar{f}, & \text { if } r \neq \pm\left(d_{1}+1\right) \\
-\frac{1}{(1 \vee|l|)}\left(\delta_{h_{r}, l_{r}} \bar{c}\right) v-\left(T_{h_{r}, l_{r}} \bar{c}\right) v_{r}+\frac{1}{(1 \vee|l|)} \xi \delta_{h_{r}, l_{r}} \bar{f}, & \text { if } r= \pm\left(d_{1}+1\right),\end{cases} \\
& I_{5 r}= \begin{cases}\sum_{p} k_{p} \frac{\left(v\left(t, x+h_{r} l_{r}+\bar{\eta}\left(x+h_{r} l_{r}, p\right)\right)-v(t, x+\bar{\eta}(x, p))\right)}{h_{r}}-\nu(E) v_{r}, & \text { if } r \neq \pm\left(d_{1}+1\right) \\
\sum_{p} k_{p} \frac{\left(v\left(t, x+h_{r} l_{r}+\bar{\eta}\left(x+h_{r} l_{r}, p\right)\right)-v(t, x+\bar{\eta}(x, p))\right)}{h_{r}(1 \vee(l))}-\nu(E) v_{r}, & \text { if } r= \pm\left(d_{1}+1\right) .\end{cases}
\end{aligned}
$$

The last term is of particular relevance to this paper as it comes from the discretization of the integral term.

Now multiply 4.10 by $\xi v_{r}^{-}$and sum up with respect to $r$. The main part of the proof involves the estimation of each of the above terms as they appear after summation had been done.

We start with the term $\sum_{r} v_{r}^{-} I_{5 r}$. Note that $v_{r}^{-} v_{r}=\left(v_{r}^{-}\right)^{2}$ and moreover that $\sum_{p} k_{p}=\nu(E)$ by $(3.2)$. We get

$$
\begin{aligned}
& \sum_{r} v_{r}^{-} I_{5 r} \\
& =\nu(E) W+\sum_{p, r \neq \pm\left(d_{1}+1\right)} k_{p} v_{r}^{-} \frac{v\left(t, x+h_{r} l_{r}+\bar{\eta}\left(x+h_{r} l_{r}, p\right)\right)-v(t, x+\bar{\eta}(x, p))}{h_{r}} \\
& +\sum_{p, r= \pm\left(d_{1}+1\right)} k_{p} v_{r}^{-} \frac{v\left(t, x+h_{r} l_{r}+\bar{\eta}\left(x+h_{r} l_{r}, p\right)\right)-v(t, x+\bar{\eta}(x, p))}{h_{r}\left(1 \vee l_{r}\right)}
\end{aligned}
$$

For $r= \pm 1, \pm 2, \ldots \ldots . . \pm d_{1}$ we have

$$
\begin{aligned}
& \left|\frac{v\left(t, x+h_{r} l_{r}+\bar{\eta}\left(x+h_{r} l_{r}, p\right)\right)-v(t, x+\bar{\eta}(x, p))}{h_{r}}\right| \\
& =\left(\frac{\epsilon}{h_{r}}\right)\left(\left|\frac{h_{r}}{\epsilon} l^{\prime}\right| \vee 1\right)\left|\frac{v\left(t, x+\bar{\eta}(x, p)+\epsilon \frac{h_{r} l^{\prime}}{\epsilon}\right)-v(t, x+\bar{\eta}(x, p))}{\epsilon\left(1 \vee\left|\frac{h_{r}}{\epsilon} l^{\prime}\right|\right)}\right| \\
& \leq K\left|l^{\prime}\right| M_{1},
\end{aligned}
$$

since $\varepsilon \leq K h_{1}$ and where

$$
l^{\prime}=l_{r}+\frac{\bar{\eta}\left(x+h_{r} l_{r}, p\right)-\bar{\eta}(x, p)}{h_{r}} \quad \text { and hence } \quad\left|l^{\prime}\right| \leq\left|l_{r}\right|+\left|l_{r}\right||\nabla \bar{\eta}(\cdot, p)|_{L^{\infty}} .
$$

For $r= \pm\left(d_{1}+1\right)$ similarly we have,

$$
\left|\frac{v(t, x+\epsilon l+\bar{\eta}(x+\epsilon l, p))-v(t, x+\bar{\eta}(x, p))}{\epsilon(1 \vee l)}\right| \leq M_{1} \frac{1 \vee\left(\left|l^{\prime}\right|\right)}{1 \vee|l|}
$$

where

$$
l^{\prime}=l+\frac{\bar{\eta}(x+\epsilon l, p)-\bar{\eta}(x, p)}{\epsilon} \quad \text { and hence } \quad\left|l^{\prime}\right| \leq|l|\left(1+|\nabla \bar{\eta}(\cdot, p)|_{L^{\infty}}\right) .
$$

Putting the above pieces together and using Cauchy-Schwartz inequality we get,

$$
\sum_{r} v_{r}^{-} I_{5 r} \geq \nu(E) W-N\left(d_{1}, K\right) \nu(E) M_{1}^{2} .
$$


Next, we estimate the term $\sum_{r} v_{r}^{-} I_{3 r}$ :

$$
\begin{aligned}
\sum_{r} v_{r}^{-} I_{3 r} & =\sum_{r} v_{r}^{-} T_{h_{r}, l_{r}} \bar{b}_{k} \delta_{h_{k}, l_{k}} v_{r}+\sum_{r \neq \pm\left(d_{1}+1\right)} v_{r}^{-}\left(\delta_{h_{r}, l_{r}} \bar{b}_{k}\right) \delta_{h_{k}, l_{k}} v \\
& +\sum_{r= \pm\left(d_{1}+1\right)} \frac{1}{1 \vee|l|} v_{r}^{-}\left(\delta_{h_{r}, l_{r}} \bar{b}_{k}\right) \delta_{h_{k}, l_{k}} v .
\end{aligned}
$$

At the maximum point $(t, x, l)$ for $V$, Lemma 4.1 and 4.1) yields for each $k$

$$
0 \geq \delta_{h_{k}, l_{k}}\left(\sum_{r}\left(v_{r}^{-}\right)^{2}\right)-\delta \delta_{h_{k}, l_{k}} C(x) \geq-2 \sum_{r} v_{r}^{-} \delta_{h_{k}, l_{k}} v_{r}-\delta \delta_{h_{k}, l_{k}} C(x),
$$

which could be rewritten as

$$
\sum_{r}\left[v_{r}^{-} \delta_{h_{k}, l_{k}} v_{r}+\frac{\delta}{4\left(d_{1}+1\right)} \delta_{h_{k}, l_{k}} C(x)\right] \geq 0 .
$$

Since $b_{k} \geq 0$, this inequality implies that

$$
\sum_{r, k} T_{h_{r}, l_{r}} \bar{b}_{k} v_{r}^{-} \delta_{h_{k}, l_{k}} v_{r} \geq-\frac{\delta}{4\left(d_{1}+1\right)} \sum_{r, k} T_{h_{r}, l_{r}} \bar{b}_{k} \delta_{h_{k}, l_{k}} C(x) .
$$

Combining this inequality with 4.12 we get the desired estimate for $\sum_{r} v_{r}^{-} I_{3 r}$,

$$
\sum_{r} v_{r}^{-} I_{3 r} \geq-\delta N\left(d_{1}, K\right)-N\left(d_{1}, K\right) M_{1}^{2} .
$$

Now we consider the term $\sum_{r} v_{r}^{-} I_{4 r}$.

$$
\begin{aligned}
\sum_{r} v_{r}^{-} I_{4 r} & =-\sum_{r \neq \pm\left(d_{1}+1\right)} v_{r}^{-}\left[\left(\delta_{h_{r}, l_{r}} \bar{c}\right) v-\left(T_{h_{r}, l_{r}} \bar{c}\right) v_{r}+\xi \delta_{h_{r}, l_{r}} \bar{f}\right] \\
& -\sum_{r= \pm\left(d_{1}+1\right)} v_{r}^{-}\left[\frac{1}{(1 \vee|l|)}\left(\delta_{h_{r}, l_{r}} \bar{c}\right) v-\left(T_{h_{r}, l_{r}} \bar{c}\right) v_{r}+\frac{1}{(1 \vee|l|)} \xi \delta_{h_{r}, l_{r}} \bar{f}\right] .
\end{aligned}
$$

We see that

$$
\sum_{r} T_{h_{r}, l_{r}} \bar{c}\left(-v_{r}\right) v_{r}^{-}=\sum_{r} T_{h_{r}, l_{r}} \bar{c}\left(v_{r}^{-}\right)^{2} \geq \lambda \sum_{r}\left(v_{r}^{-}\right)^{2}=\lambda W .
$$

Young's inequality and the definition of $M$ then gives

$$
\sum_{r} v_{r}^{-} I_{4 r} \geq-N\left(K, d_{1}\right) M_{1}\left(e^{c_{0} T^{\prime}}+M\right)+\lambda W .
$$

Consider the $\sum_{r} v_{r}^{-} \delta_{\tau}^{T}\left(\xi^{-1} v\right)$ term. Once more using that $(t, x, l)$ is a maximum point of $V$, Lemma 4.1, and 4.1), we get

$$
0 \leq-\delta_{\tau}^{T}\left(\sum_{r}\left(v_{r}^{-}\right)^{2}\right)=-2 \sum_{r} v_{r}^{-} \delta_{\tau}^{T}\left(v_{r}^{-}\right)-\tau \sum_{r}\left(\delta_{\tau}^{T}\left(v_{r}^{-}\right)\right)^{2} \leq 2 \sum_{r} v_{r}^{-} \delta_{\tau}^{T}\left(v_{r}\right) .
$$

We conclude that

$$
\begin{aligned}
\sum_{r} \xi v_{r}^{-} \delta_{\tau}^{T}\left(\xi^{-1} v_{r}\right) & =\sum_{r} \xi v_{r}^{-}\left[\xi^{-1}\left(t+\tau_{T}(t)\right) \delta_{\tau}^{T} v_{r}+v_{r} \delta_{\tau}^{T} \xi^{-1}\right] \\
& =e^{-c_{0} \tau_{T}(t)} \sum_{r} v_{r}^{-} \delta_{\tau}^{T} v_{r}-W \xi \delta_{\tau} \xi^{-1} \\
& \geq W \frac{1-e^{-c_{0} \tau}}{\tau}
\end{aligned}
$$


Using the relations 4.15, 4.14, 4.13, and 4.11, we obtain from 4.10

$$
\begin{aligned}
& W\left[\frac{1-e^{-c_{0} \tau}}{\tau}+\lambda\right]+\nu(E) V-\nu(E) N\left(d_{1}, K\right) M_{1}^{2}-\delta N\left(d_{1}, K\right)-N\left(d_{1}, K\right) M_{1}^{2} \\
& -N\left(d_{1}, K\right) M_{1}\left(e^{c_{0} T^{\prime}}+M\right)+\sum_{r, k} v_{r}^{-}\left[\bar{a}_{k} \Delta_{h_{k}, l_{k}} v_{r}+I_{1 r}+I_{2 r}\right] \leq 0,
\end{aligned}
$$

i.e.,

$$
\begin{aligned}
& W\left[\frac{1-e^{-c_{0} \tau}}{\tau}+\lambda\right]+\nu(E) V-\nu(E) N\left(d_{1}, K\right) M_{1}^{2}-\delta N\left(d_{1}, K\right) \\
& \quad-N\left(d_{1}, K\right) M_{1}^{2}-N\left(d_{1}, K\right) M_{1}\left(e^{c_{0} T^{\prime}}+M\right) \\
& \leq-\sum_{r, k} v_{r}^{-} \bar{a}_{k} \Delta_{h_{k}, l_{k}} v_{r}-\sum_{k, r \neq \pm\left(d_{1}+1\right)} v_{r}^{-}\left(\delta_{h_{r}, l_{r}} \bar{a}_{k}\right) \Delta_{h_{k}, l_{k}} v \\
& \quad-\sum_{k, r= \pm\left(d_{1}+1\right)} \frac{1}{1 \vee|l|} v_{r}^{-}\left(\delta_{h_{r}, l_{r}} \bar{a}_{k}\right) \Delta_{h_{k}, l_{k}} v-\sum_{r, k} h_{r} v_{r}^{-}\left(\delta_{h_{r}, l_{r}} \bar{a}_{k}\right) \Delta_{h_{k}, l_{k}} v_{r} .
\end{aligned}
$$

Once again, using the fact that $(t, x, l)$ is a point of maxima for $V$, along with the discrete product rule (Lemma 4.1) and (4.1), we have for each $k$,

$$
\begin{aligned}
0 & \geq \Delta_{h_{k}, l_{k}}\left(\sum_{r}\left(v_{r}^{-}\right)^{2}\right)-\delta \Delta_{h_{k}, l_{k}} C(x) \\
& =2 \sum_{r} v_{r}^{-} \Delta_{h_{k}, l_{k}} v_{r}^{-}+\sum_{r}\left[\left(\delta_{h_{k}, l_{k}} v_{r}^{-}\right)^{2}+\left(\delta_{h_{k}, l_{-k}} v_{r}^{-}\right)^{2}\right]-\delta \Delta_{h_{k}, l_{k}} C(x) \\
& \geq-2 \sum_{r} v_{r}^{-} \Delta_{h_{k}, l_{k}} v_{r}+\sum_{r}\left[\left(\delta_{h_{k}, l_{k}} v_{r}^{-}\right)^{2}+\left(\delta_{h_{k}, l_{-k}} v_{r}^{-}\right)^{2}\right]-\delta \Delta_{h_{k}, l_{k}} C(x) .
\end{aligned}
$$

We rewrite this as

$$
2 \sum_{r} v_{r}^{-} \Delta_{h_{k}, l_{k}} v_{r}+\delta \Delta_{h_{k}, l_{k}} C(x) \geq \sum_{r}\left[\left(\delta_{h_{k}, l_{k}} v_{r}^{-}\right)^{2}+\left(\delta_{h_{k}, l_{-k}} v_{r}^{-}\right)^{2}\right],
$$

and conclude that

$$
2 \sum_{r} v_{r}^{-} \Delta_{h_{k}, l_{k}} v_{r}+\delta \Delta_{h_{k}, l_{k}} C(x) \geq 0 .
$$

Multiplying 4.17) by $\bar{a}_{k}$ and summing up with respect to $k$ we get

$$
\sum_{r, k} v_{r}^{-} \bar{a}_{k} \Delta_{h_{k}, l_{k}} v_{r}+\sum_{k} \frac{\delta}{2} \bar{a}_{k} \Delta_{h_{k}, l_{k}} C(x) \geq \sum_{r, k} \bar{a}_{k}\left(\delta_{h_{k}, l_{k}}\left(v_{r}^{-}\right)\right)^{2} .
$$

Using this inequality, 4.16 becomes

$$
\begin{aligned}
& W\left[\frac{1-e^{-c_{0} \tau}}{\tau}+\lambda\right]+\nu(E) V-\nu(E) N\left(d_{1}, K\right) M_{1}^{2}-\delta N\left(d_{1}, K\right) \\
& \quad-N\left(d_{1}, K\right) M_{1}^{2}-N\left(d_{1}, K\right) M_{1}\left(e^{c_{0} T^{\prime}}+M\right) \\
& \leq J_{1}+J_{2}+\frac{\delta}{4} \sum_{k} \bar{a}_{k} \Delta_{h_{k}, l_{k}} C
\end{aligned}
$$

where

$$
J_{1}=\sum_{r, k} v_{r}^{-}\left|\left(\delta_{h_{r}, l_{r}} \bar{a}_{k}\right) \Delta_{h_{k}, l_{k}} v\right|-\frac{1}{4} \sum_{r, k} \bar{a}_{k}\left(\delta_{h_{k}, l_{k}} v_{r}^{-}\right)^{2}
$$




$$
J_{2}=\sum_{r, k} v_{r}^{-} h_{r}\left|\left(\delta_{h_{r}, l_{r}} \bar{a}_{k}\right) \Delta_{h_{k}, l_{k}} v_{r}\right|-\frac{1}{2} \sum_{r, k} v_{r}^{-} \bar{a}_{k} \Delta_{h_{k}, l_{k}} v_{r}-\frac{1}{4} \sum_{r, k} \bar{a}_{k}\left(\delta_{h_{k}, l_{k}} v_{r}^{-}\right)^{2} .
$$

Now we estimate $J_{1}$. By 4.2 ,

$$
\left|\Delta_{h_{k}, l_{k}} v\right| \leq \sum_{r}\left|\delta_{h_{k}, l_{k}} v_{r}^{-}\right|+\sum_{r}\left|\delta_{h_{k}, l_{-k}} v_{r}^{-}\right|,
$$

and by Lemma 4.1 and Young's inequality, we get

$$
\begin{aligned}
& \sum_{r, k} v_{r}^{-}\left|\left(\delta_{h_{r}, l_{r}}\left(\bar{\sigma}_{k}\right)^{2}\right) \Delta_{h_{k}, l_{k}} v\right|=\sum_{r, k} v_{r}^{-}\left|\left(2 \bar{\sigma}_{k} \delta_{h_{r}, l_{r}} \bar{\sigma}_{k}+h_{r}\left(\delta_{h_{r}, l_{r}} \bar{\sigma}_{k}\right)^{2}\right) \Delta_{h_{k}, l_{k}} v\right| \\
& \leq \sum_{r, k} M_{1} K\left|\bar{\sigma}_{k} \Delta_{h_{k}, l_{k}} v\right|+K^{3} 2\left(d_{1}+1\right) M_{1} \sum_{k} h_{1}\left|\Delta_{h_{k}, l_{k}} v\right| \\
& \leq N M_{1} \sum_{k}\left|\bar{\sigma}_{k} \Delta_{h_{k}, l_{k}} v\right|+N M_{1}^{2} \stackrel{4.20}{\leq} N \sum_{r, k} M_{1}\left|\bar{\sigma}_{k} \delta_{h_{k}, l_{k}} v_{r}^{-}\right|+N M_{1}^{2} \\
& \leq N \sum_{r, k}\left(8 N M_{1}^{2}+\frac{1}{8 N}\left|\bar{\sigma}_{k} \delta_{h_{k}, l_{k}} v_{r}^{-}\right|^{2}\right)+N M_{1}^{2} \leq \frac{1}{4} \sum_{r, k} \bar{a}_{k}\left(\delta_{h_{k}, l_{k}} v_{r}^{-}\right)^{2}+N M_{1}^{2},
\end{aligned}
$$

which implies that $J_{1} \leq N M_{1}^{2}$.

The next step is to get a similar estimate on $J_{2}$. Note that

$$
|a|=2 a_{-}+a, \quad h_{r} \leq K h_{1}, \quad h_{r}^{2}\left|\Delta_{h_{k}, l_{k}} v_{r}\right| \leq M_{1} .
$$

We get

$$
\begin{aligned}
& \sum_{r, k} v_{r}^{-} h_{r}\left|\left(\delta_{h_{r}, l_{r}} \bar{a}_{k}\right) \Delta_{h_{k}, l_{k}} v_{r}\right| \\
& \leq \sum_{r, k} v_{r}^{-} h_{r}\left|2\left(\delta_{h_{r}, l_{r}} \bar{\sigma}_{k}\right) \bar{\sigma}_{k} \Delta_{h_{k}, l_{k}} v_{r}+h_{r}\left(\delta_{h_{r}, l_{r}} \bar{\sigma}_{k}\right)^{2} \Delta_{h_{k}, l_{k}} v_{r}\right| \\
& \leq \sum_{r, k} N_{1}\left|v_{r}^{-} h_{r} \bar{\sigma}_{k} \Delta_{h_{k}, l_{k}} v_{r}\right|+\sum_{r, k} N_{2} h_{r}^{2} v_{r}^{-}\left|\Delta_{h_{k}, l_{k}} v_{r}\right| \\
& \leq \sum_{r, k} 2 N_{1} h_{r} v_{r}^{-}\left|\bar{\sigma}_{k}\right|\left(\Delta_{h_{k}, l_{k}} v_{r}\right)_{-}+\sum_{r, k} N_{1} h_{r} v_{r}^{-}\left|\bar{\sigma}_{k}\right| \Delta_{h_{k}, l_{k}} v_{r}+N_{2} M_{1}^{2} .
\end{aligned}
$$

In the above inequality the summation over $r$ may be restricted to the cases where $v_{r}^{-} \neq 0$ or $v_{r}<0$. From 4.1 and $h_{k} \Delta_{h_{k}, l_{k}}=\delta_{h_{k}, l_{k}}+\delta_{h_{k},-l_{k}}$, we then get

$$
h_{k}\left(\Delta_{h_{k}, l_{k}} v_{r}\right)_{-}=h_{k} \max \left(-\Delta_{h_{k}, l_{k}} v_{r}, 0\right) \leq h_{k}\left|\Delta_{h_{k}, l_{k}} v_{r}^{-}\right| \leq\left|\delta_{h_{k}, l_{k}} v_{r}^{-}\right|+\left|\delta_{h_{k}, l_{-k}} v_{r}^{-}\right| \text {. }
$$

The last two estimates give

$$
\begin{aligned}
& \sum_{r, k} v_{r}^{-} h_{r}\left|\left(\delta_{h_{r}, l_{r}} \bar{a}_{k}\right) \Delta_{h_{k}, l_{k}} v_{r}\right| \\
& \quad \leq N_{2} M_{1}^{2}+\sum_{r, k}\left(\frac{1}{4} \bar{a}_{k}\left(\delta_{h_{k}, l_{k}} v_{r}^{-}\right)^{2}+N_{1} v_{r}^{-} h\left|\bar{\sigma}_{k}\right| \Delta_{h_{k}, l_{k}} v_{r}\right),
\end{aligned}
$$

and hence

$$
J_{2} \leq N_{2} M_{1}^{2}-\frac{1}{2}\left(\bar{a}_{k}-2 N_{1} h \sqrt{\bar{a}_{k}}\right) v_{r}^{-} \Delta_{h_{k}, l_{k}} v_{r}
$$

Let

$$
\mathbb{A}=\left\{k:\left(\bar{a}_{k}-2 N_{1}(K) h_{1} \sqrt{\overline{a_{k}}}\right) \geq 0\right\} .
$$


and note that if $k \notin \mathbb{A}$, then

$$
\sqrt{\overline{a_{k}}} \leq 2 N_{1}(K) h_{1}, \quad \bar{a}_{k} \leq 4 N_{1}^{2} h_{1}^{2}, \quad\left|\bar{a}_{k}-2 N_{1}(K) h_{1} \sqrt{\overline{a_{k}}}\right| \leq N(K) h_{1}^{2} .
$$

By 4.18 we then get

$$
\begin{aligned}
& -\frac{1}{2} \sum_{r, k}\left(\bar{a}_{k}-2 N_{2}(K) h_{1} \sqrt{\overline{a_{k}}}\right) v_{r}^{-} \Delta_{h_{k}, l_{k}} v_{r} \\
& =-\frac{1}{2}\left(\sum_{r, k \in \mathbb{A}}+\sum_{r, k \notin \mathbb{A}}\right)\left(\bar{a}_{k}-2 N_{2}(K) h_{1} \sqrt{\bar{a}_{k}}\right) v_{r}^{-} \Delta_{h_{k}, l_{k}} v_{r} \\
& \leq-\frac{1}{2} \sum_{r, k \in \mathbb{A}}\left(\bar{a}_{k}-2 N_{2}(K) h_{1} \sqrt{\bar{a}_{k}}\right) v_{r}^{-} \Delta_{h_{k}, l_{k}} v_{r}+N(K) \sum_{r, k \notin \mathbb{A}} v_{r}^{-} h_{1}^{2} \Delta_{h_{k}, l_{k}} v_{r} \\
& \left.\leq \frac{1}{2} \delta \sum_{r, k \in \mathbb{A}}\left(\bar{a}_{k}-2 N_{2}(K) h_{1} \sqrt{\bar{a}_{k}}\right) \Delta_{h_{k}, l_{k}} C(x)+N\left(d_{1}, K\right) M_{1}^{2}\right] \\
& \leq N\left(K, d_{1}\right)\left(M_{1}^{2}+\delta\right),
\end{aligned}
$$

which gives the estimate $J_{2} \leq N\left(M_{1}^{2}+\delta\right)$.

The bounds on $J_{1}$ and $J_{2}$ along with 4.19) and the definition of $V(V=W-\delta C)$ give

$$
\left(\lambda+\frac{1-e^{-c_{0} \tau}}{\tau}\right) V(t, x, l) \leq N\left(\delta+\left(e^{c_{0} T^{\prime}}+M_{1}+M\right) M_{1}\right),
$$

when $t<T$. Combining this estimate with the estimate for $t=T 4.5$ we see that $\left.V(t, x, l) \leq\left(\lambda+\frac{1-e^{-c_{0} \tau}}{\tau}\right)^{-1} N\left(\delta+e^{c_{0} T^{\prime}}+M_{1}+M\right) M_{1}+N e^{2 c_{0} T^{\prime}} \sup _{r, x} u_{r}(T, x)\right)^{2}$, for every $(t, x, l) \in \overline{\mathcal{M}}_{T} \times \mathbb{R}^{N}$ and $\delta>0$. Using the definition of $V$ and sending $\delta \rightarrow 0$ then give for every $t, x, l$,

$$
\begin{aligned}
& W(t, x, l) \\
& \leq\left(\lambda+\frac{1-e^{-c_{0} \tau}}{\tau}\right)^{-1} N\left(e^{c_{0} T^{\prime}}+M_{1}+M\right) M_{1}+N e^{2 c_{0} T^{\prime}}\left(\sup _{r, x} u_{r}(T, x)\right)^{2} .
\end{aligned}
$$

Let $W_{\max }=\sup _{(t, x, l) \in \overline{\mathcal{M}}_{T} \times \mathbb{R}^{d}} W(t, x, l)$. For each $(t, x) \in \overline{\mathcal{M}}_{T}$ and for each $r$, either $v_{r}(t, x) \leq 0$ or $-v_{r}(t, x)=v_{-r}\left(t, x+h_{r} l_{r}\right) \leq 0$. In any case we have $\left|v_{r}(t, x)\right| \leq \sqrt{W_{\max }}$ and hence

$$
M_{1} \leq \sqrt{W_{\max }} \quad \text { and } \quad \frac{1}{1 \vee|l|}\left|\delta_{\epsilon, \pm l} u\right| \leq \sqrt{W_{\max }} .
$$

In view of 4.21

$$
\begin{aligned}
W_{\max } \leq(\lambda+ & \left.\frac{1-e^{-c_{0} \tau}}{\tau}\right)^{-1} N\left(e^{c_{0} T^{\prime}}+\sqrt{W_{\max }}+M\right) \sqrt{W_{\max }} \\
& +N e^{2 c_{0} T^{\prime}}\left(\sup _{r, x} u_{r}(T, x)\right)^{2} .
\end{aligned}
$$

By this estimate, Young's inequality, and $M \leq e^{c_{0} T^{\prime}}|u|_{0}$, we obtain

$$
W_{\max } \leq\left(\lambda+\frac{1-e^{-c_{0} \tau}}{\tau}\right)^{-1} N\left(1+W_{\max }+|u|_{0}^{2}\right)+N\left(\sup _{r, x} u_{r}(T, x)\right)^{2} .
$$


If $\left(\lambda+\frac{1-e^{-c_{0} \tau}}{\tau}\right) \geq N+1$, then we conclude that

$$
W_{\max } \leq N^{\prime}\left[1+M_{0}^{2}+\left(\sup _{r, x} u_{r}(T, x)\right)^{2}\right] .
$$

Along with 4.22 this estimate proves the theorem.

Next, following 21, we prove a continuous dependence estimate for the scheme. Let $\hat{\sigma}_{k}^{\alpha}, \hat{b}_{k}^{\alpha}, \hat{c}^{\alpha}, \hat{f}^{\alpha}, \hat{u}_{0}^{\alpha}, \hat{\eta}^{\alpha}$ be functions from $\mathcal{A} \times \mathbb{R} \times \mathbb{R}^{d}$ to $\mathbb{R}$ and set $\hat{a}_{k}^{\alpha}=\frac{1}{2}\left|\hat{\sigma}_{k}^{\alpha}\right|^{2}$.

Theorem 4.4. Assume (A.1), (A.3), (A.2), (3.1) and (3.2) hold. Let $\hat{\sigma}_{\underline{k}}^{\alpha}, \hat{b}_{k}^{\alpha}, \hat{c}^{\alpha}, \hat{f}^{\alpha}, \hat{\eta}^{\alpha}$ satisfy assumptions (A.1) - (A.3) Let $u$ and $\hat{u}$ be functions on $\overline{\mathcal{M}}_{T}$ satisfying (3.3) with coefficients $\sigma_{k}^{\alpha}, b_{k}^{\alpha}, c^{\alpha}, f^{\alpha}, \eta^{\alpha}$ and $\hat{\sigma}_{k}^{\alpha}, \hat{b}_{k}^{\alpha}, \hat{c}^{\alpha}, \hat{f}^{\alpha}, \hat{\eta}^{\alpha}$ respectively and $|u(T, \cdot)|_{1}+|\hat{u}(T, \cdot)|_{1} \leq K$. Define

$$
\epsilon:=\sup _{\overline{\mathcal{M}}_{T}, A, k}\left\{\left|\hat{\sigma}_{k}^{\alpha}-\sigma_{k}^{\alpha}\right|+\left|\hat{b}_{k}^{\alpha}-b_{k}^{\alpha}\right|+\left|\hat{c}^{\alpha}-c^{\alpha}\right|+\left|\hat{f}^{\alpha}-f^{\alpha}\right|+\left|\hat{\eta}^{\alpha}-\eta^{\alpha}\right|\right\} .
$$

Then if there exists a $c_{0} \geq 0$ satisfying (4.4), there exists a constant $N$ depending only on $K, d_{1}, \lambda, c_{0}, T, \nu(E)$ such that

$$
|u-\hat{u}| \leq N I \epsilon \quad \text { on } \overline{\mathcal{M}}_{T},
$$

where

$$
I:=1+\max _{k}\left|\delta_{h_{1}, l_{k}} u(T, \cdot)\right|_{0}+\max _{k}\left|\delta_{h_{1}, l_{k}} \hat{u}(T, \cdot)\right|_{0}+\epsilon^{-1}|u(T, \cdot)-\hat{u}(T, \cdot)|_{0} .
$$

Proof. First we show that it is sufficient to prove the result assuming $\epsilon \leq h_{1}$. For each $\theta \in[0,1]$, let $u^{\theta}$ be the (unique) solution of

$$
\begin{aligned}
\delta_{\tau}^{T} u & +\sup _{\alpha \in \mathcal{A}}\left[a_{k}^{\theta \alpha} \Delta_{h_{1}, l_{k}} u+b_{k}^{\theta \alpha} \delta_{h_{k}, l_{k}} u-c^{\theta \alpha} u+f^{\theta \alpha}\right. \\
& \left.+\sum_{p} k_{p}\left(u\left(t, x+\eta^{\theta \alpha}(x, p)\right)-u(t, x)\right)\right]=0 \text { in } \mathcal{M}_{T},
\end{aligned}
$$

with $u^{\theta}(T, x)=(1-\theta) u(T, x)+\theta \hat{u}(T, x)$ and where

$$
\left[\sigma_{k}^{\theta \alpha}, b_{k}^{\theta \alpha}, c^{\theta \alpha}, f^{\theta \alpha}, \eta^{\theta \alpha}\right]=(1-\theta)\left[\sigma_{k}^{\alpha}, b_{k}^{\alpha}, c^{\alpha}, f^{\alpha}, \eta^{\alpha}\right]+\theta\left[\hat{\sigma}_{k}^{\alpha}, \hat{b}_{k}^{\alpha}, \hat{c}^{\alpha}, \hat{f}^{\alpha}, \hat{\eta}^{\alpha}\right] .
$$

By uniqueness, $u^{0}=u$ and $u^{1}=\hat{u}$. Also note that for any $\theta_{1}, \theta_{2} \in[0,1], \alpha, k$,

$$
\begin{aligned}
& \left|\sigma_{k}^{\theta_{1} \alpha}-\sigma_{k}^{\theta_{2} \alpha}\right|_{0}+\left|b_{k}^{\theta_{1} \alpha}-b_{k}^{\theta_{2} \alpha}\right|_{0}+\left|c^{\theta_{1} \alpha}-c^{\theta_{2} \alpha}\right|_{0}+\left|f^{\theta_{1} \alpha}-f^{\theta_{2} \alpha}\right|_{0}+\left|\eta^{\theta_{1} \alpha}-\eta^{\theta_{2} \alpha}\right|_{0} \\
& \leq\left|\theta_{1}-\theta_{2}\right| \epsilon .
\end{aligned}
$$

Therefore if we assume the result holds for $\epsilon \leq h_{1}$, then for any $\epsilon$ satisfying $\mid \theta_{1}-$ $\theta_{2} \mid \epsilon \leq h_{1}$, we have

$$
\left|u^{\theta_{1}}-u^{\theta_{2}}\right| \leq N_{1}\left|\theta_{1}-\theta_{2}\right| \epsilon I\left(\theta_{1}, \theta_{2}\right)
$$

where

$$
\begin{aligned}
I\left(\theta_{1}, \theta_{2}\right) & =1+\max _{k}\left|\delta_{h_{1}, l_{k}} u^{\theta_{1}}(T, \cdot)\right|_{0}+\max _{k}\left|\delta_{h_{1}, l_{k}} u^{\theta_{2}}(T, \cdot)\right|_{0} \\
& +\epsilon^{-1}\left|\theta_{1}-\theta_{2}\right|^{-1}\left|u^{\theta_{1}}(T, \cdot)-u^{\theta_{2}}(T, \cdot)\right|_{0} .
\end{aligned}
$$

Clearly $I\left(\theta_{1}, \theta_{2}\right) \leq 4 I$, so by dividing the interval $[0,1]$ into sufficient number of sub-intervals $\theta_{1}, \ldots, \theta_{n}$, we can conclude the theorem (with $4 N$ instead of $N$ ), by writing

$$
u-\hat{u}=\sum_{i=1}^{n}\left(u^{\theta_{i}}-u^{\theta_{i-1}}\right)
$$


and using 4.24 to estimate each $u^{\theta_{i}}-u^{\theta_{i-1}}$. Henceforth we assume that $\epsilon \leq h_{1}$.

We will now show that the continuous dependence estimate 4.23 is a consequence of the Lipschitz estimate Theorem (4.3). To this end, we consider $\mathbb{R}^{d}$ as subspace of $\mathbb{R}^{d+1}$ and write,

$$
\begin{gathered}
\mathbb{R}^{d+1}=\left\{\left(x^{\prime}, x^{d+1}\right): x^{\prime} \in \mathbb{R}^{d}, x^{d+1} \in \mathbb{R}\right\}, \\
\bar{Q}_{T}(d+1)=[0, T] \times \mathbb{R}^{d+1}, \quad \overline{\mathcal{M}}_{T}(d+1)=\{j \tau \wedge T: j=0,1,2 \ldots . .\} \times \mathbb{R}^{d+1} \\
Q_{T}(d+1)=[0, T) \times \mathbb{R}^{d+1}, \quad \text { and } \quad \mathcal{M}_{T}(d+1)=Q_{T}(d+1) \cap \overline{\mathcal{M}}_{T}(d+1) .
\end{gathered}
$$

Let $\rho \in C^{1}(\mathbb{R})$ be a bounded function on $\mathbb{R}$ such that

$$
\rho(-1)=1, \rho(0)=0, \rho^{\prime}(p)=\rho^{\prime}(q)=0 \text { if } p \leq-1, q \geq 0 .
$$

Now define

$$
\tilde{\sigma}_{k}^{\alpha}\left(t, x^{\prime}, x_{d+1}\right):=\hat{\sigma}_{k}^{\alpha}\left(t, x^{\prime}\right) \rho\left(\frac{x^{d+1}}{\epsilon}\right)+\sigma_{k}^{\alpha}\left(t, x^{\prime}\right)\left(1-\rho\left(\frac{x^{d+1}}{\epsilon}\right)\right),
$$

and in a similar way, $\tilde{b}_{k}^{\alpha}, \tilde{c}_{k}^{\alpha}, \tilde{f}_{k}^{\alpha}, \tilde{u}(T, \cdot)$. Define $\tilde{\eta}^{\alpha}: \mathbb{R}^{d+1} \times \mathbb{R}^{M} \mapsto \mathbb{R}^{d+1}$ as

$$
\tilde{\eta}^{\alpha}\left(x^{\prime}, x_{d+1} ; z\right)=\left(\hat{\eta}^{\alpha}\left(x^{\prime}, z\right), 0\right) \rho\left(\frac{x^{d+1}}{\epsilon}\right)+\left(\eta^{\alpha}\left(x^{\prime}, z\right), 0\right)\left(1-\rho\left(\frac{x^{d+1}}{\epsilon}\right)\right) .
$$

We would like to show that, $\tilde{\sigma}_{k}^{\alpha}, \tilde{b}_{k}^{\alpha}, \tilde{c}_{k}^{\alpha}, \tilde{f}_{k}^{\alpha}, \tilde{\eta}_{k}^{\alpha}$ and $\tilde{u}(T, \cdot)$ all satisfy the assumptions $(\mathbf{A} .1)$ and (A.3) in $\bar{Q}_{T}(d+1)$. All other properties apart from Lipschitz continuity in $(d+1)$-th direction are straight forward. For $\tilde{\sigma}_{k}^{\alpha}$ along $(d+1)$-direction we have

$$
\left|\frac{\partial \tilde{\sigma}_{k}^{\alpha}}{\partial x^{d+1}}\right|=\frac{1}{\epsilon}\left|\hat{\sigma}_{k}^{\alpha}\left(t, x^{\prime}\right)-\sigma_{k}^{\alpha}\left(t, x^{\prime}\right)\right|\left|\rho^{\prime}\left(\frac{x^{d+1}}{\epsilon}\right)\right| \leq\left|\rho^{\prime}\left(\frac{x^{d+1}}{\epsilon}\right)\right| \leq K .
$$

A similar conclusion holds for the other functions.

Therefore by Lemma 3.1 there exists a function $\tilde{u}_{\tau, h}$, defined on $\overline{\mathcal{M}}_{T}(d+1)$, which solves 3.3 with the new family of coefficients $\tilde{\sigma}_{k}^{\alpha}, \tilde{b}_{k}^{\alpha}, \tilde{c}_{k}^{\alpha}, \tilde{f}_{k}^{\alpha}, \tilde{\eta}^{\alpha}$ and terminal data $\tilde{u}(T, \cdot)$. Furthermore, by uniqueness, we must have

$$
\tilde{u}_{\tau, h}\left(t, x^{\prime},-\epsilon\right)=\hat{u}\left(t, x^{\prime}\right), \quad \tilde{u}_{\tau, h}\left(t, x^{\prime}, 0\right)=\tilde{u}_{\tau, h}\left(t, x^{\prime}, \varepsilon\right)=u\left(t, x^{\prime}\right) .
$$

Now we choose $l=(0,0, \ldots, 1)$, the unit vector along the $(d+1)$-th direction. For this $l$, by Theorem 4.3 (since $\varepsilon \leq h_{1}$ ) we conclude that there exists a constant $N$, depending only on $d, d_{1}, K, c_{0}, \lambda, T$, and $\nu$, such that

$$
\left|\frac{\tilde{u}_{\tau, h}\left(t, x^{\prime},-\epsilon\right)-\tilde{u}_{\tau, h}\left(t, x^{\prime}, 0\right)}{\epsilon}\right| \leq N \sup _{k, x, l}\left[1+\left|\delta_{h_{1}, l_{k}} \tilde{u}(T, x)\right|+\frac{1}{1 \vee|l|}\left|\delta_{\epsilon, \pm l} \tilde{u}(T, x)\right|\right]
$$

which gives $|\hat{u}-u| \leq N I \epsilon$.

Next we establish Lipschitz continuity property of $v_{\tau, h}$ in the $x$-variable. To do this, let $S \subset B_{1}=\left\{x \in \mathbb{R}^{d}:|x|<1\right\}$ be nonempty, $\epsilon \in \mathbb{R}$, and $v_{\tau, h}^{\epsilon, S}$ be the unique solution of the equation

$$
\begin{aligned}
& \delta_{\tau}^{T} u+\sup _{(\alpha, y) \in \mathcal{A} \times S}\left[L_{h_{1}}^{\alpha}(t, x+\epsilon y) u(t, x)+f^{\alpha}(t, x+\epsilon y)\right. \\
& \left.\quad+\sum_{p} k_{p}\left(u\left(t, x+\eta^{\alpha}(x+\epsilon y, p)\right)-u(t, x)\right)\right]=0 \quad \text { in } Q_{T},
\end{aligned}
$$

with the terminal data

$$
u(T, x)=\sup _{y \in S} u_{0}(x+\epsilon y) .
$$


Note that if $S$ is a singleton $\{y\}$, then by uniqueness $v_{\tau, h}^{\epsilon, S}(t, x)=v_{\tau, h}(t, x+\epsilon y)$.

Lemma 4.5. Assume (A.1), (A.3), (A.2), and (3.1), (3.2) hold. There is a constant $N$ depending only on $K, d_{1}, \nu(E), T$, so that if the condition 4.4 is satisfied for a constant $c_{0} \geq 0$, then for every $\epsilon \in \mathbb{R}$,

$$
\left|v_{\tau, h}^{\epsilon, S}-v_{\tau, h}\right| \leq N_{1}|\epsilon| \quad \text { on } \quad \bar{Q}_{T},
$$

where $N_{1}$ only depend on $K, d_{1}, \nu(E), T, \lambda, c_{0}$. Furthermore, by choosing $S:=$ $\left\{\frac{(y-x)}{|y-x|}\right\}, \epsilon=|y-x|$, we have

$$
\left|v_{\tau, h}(t, x)-v_{\tau, h}(t, y)\right| \leq N_{1}|y-x| \quad \text { for all } \quad(t, y),(t, x) \in \bar{Q}_{T} .
$$

Proof. It is sufficient to prove 4.26) on $\overline{\mathcal{M}}_{T}$. We use of Theorem 4.4 , where we choose $A \times S,(\sigma, b, c, f, \eta)$ and $(\sigma, b, c, f, \eta)(x+\epsilon y, t / z)$ in places of $A,(\sigma, b, c, f, \eta)$ and $\hat{\sigma}, \hat{b}, \hat{c}, \hat{f}, \hat{\eta}$, respectively. The contribution from the difference of the terminal data can be bounded by $N \epsilon$.

A step in the direction of establishing Theorem 3.4 is to prove

Lemma 4.6. Assume (A.1), (A.2), (A.3) and (3.1), (3.2) hold. Let $h_{1}, h_{2}, \tau \leq K$. Let $\left(s_{0}, x_{0}\right) \in \overline{\mathcal{M}}_{T}$ and set

$$
L:=\sup _{x \in \mathbb{R}^{N}, x \neq x_{0}} \frac{\left|v_{\tau, h}\left(s_{0}, x\right)-v_{\tau, h}\left(s_{0}, x_{0}\right)\right|}{\left|x-x_{0}\right|} .
$$

Then for all $\left(t_{0}, x_{0}\right) \in \mathcal{M}_{T}$ satisfying $s_{0}-1 \leq t_{0} \leq s_{0}$ and $\frac{1}{\tau} T \in \mathbb{N}$, we have

$$
\left|v_{\tau, h}\left(s_{0}, x_{0}\right)-v_{\tau, h}\left(t_{0}, x_{0}\right)\right| \leq N(L+1)\left|s_{0}-t_{0}\right|^{\frac{1}{2}},
$$

where $N$ depends only on $K, d_{1}$ and $\nu(E)$.

Proof. Without loss of generality we may restrict ourselves to the case $0<s_{0}<1$ and $t_{0}=0$. This claim follows by shifting the origin and observing that there holds $\left|v_{\tau, h}\left(s_{0}, x_{0}\right)-v_{\tau, h}\left(t_{0}, x_{0}\right)\right| \leq 2\left|v_{\tau, h}\right|_{0}\left|s_{0}-t_{0}\right|^{\frac{1}{2}}$ whenever $\left|t_{0}-s_{0}\right|>1$.

Fix a constant $\gamma>0$. We are going to work with $\overline{\mathcal{M}}_{s_{0}}$. On $\overline{\mathcal{M}}_{s_{0}}$ we define

$$
\psi=\gamma L\left[\zeta+\kappa\left(s_{0}-t\right)\right]+K\left(s_{0}-t\right)+\gamma^{-1} L+v_{\tau, h}\left(s_{0}, x_{0}\right),
$$

where

$$
\xi(t)=e^{s_{0}-t}, \quad \beta(x)=\left|x-x_{0}\right|^{2}, \quad \zeta=\xi \eta,
$$

and the constant $\kappa$ will be chosen later. We will show that if $\kappa$ is big enough, then $\psi$ is a supersolution of 3.3 .

On $M_{s_{0}}$ we have, $\delta_{\tau}^{s_{o}} \xi=-\theta \xi$ where, $\theta:=\tau^{-1}\left(1-e^{-\tau}\right) \geq K^{-1}\left(1-e^{-K}\right)$. Furthermore, using (A.1) (A.3) we get

$$
\begin{aligned}
& \mathcal{L}_{h_{1}}^{\alpha} \beta(t, x)+\sum_{p} k_{p}\left[\beta\left(t, x+\eta^{\alpha}(x, p)\right)-\beta(t, x)\right] \\
& =2 a_{k}^{\alpha}(t, x)\left|l_{k}\right|^{2}+b_{k}^{\alpha}(t, x)\left(l_{k}, 2\left(x-x_{0}\right)+h_{1} l_{k}\right)-c^{\alpha} \beta(t, x) \\
& \quad+\sum_{p} k_{p}\left\langle\eta^{\alpha}(x, p) ; 2\left(x-x_{0}\right)+\eta^{\alpha}(x, p)\right\rangle \\
& \leq N_{1}\left(d_{1}, K, \nu(E)\right)\left(1+\left|x-x_{0}\right|\right),
\end{aligned}
$$


and hence

$$
\begin{aligned}
& \delta_{\tau}^{s_{0}} \zeta(t, x)+\mathcal{L}_{h_{1}}^{\alpha} \zeta(t, x)+\sum_{p} k_{p}\left[\zeta\left(t, x+\eta^{\alpha}(x, p)\right)-\zeta(t, x)\right] \\
& \leq N\left(d_{1}, K, \nu(E)\right)\left(1+\left|x-x_{0}\right|\right)-\theta(\tau)\left|x-x_{0}\right|^{2}
\end{aligned}
$$

Applying the same operator on $\psi$ and using the above estimates, we have

$$
\begin{aligned}
& \delta_{\tau}^{s_{0}} \psi+\mathcal{L}_{h_{1}}^{\alpha} \psi+f^{\alpha}+\sum_{p} k_{p}\left[\psi\left(t, x+\eta^{\alpha}(x, p)\right)-\psi(t, x)\right] \\
& \leq \gamma L\left[N_{2}\left(1+\left|x-x_{0}\right|\right)-\theta\left|x-x_{0}\right|^{2}-\kappa\right]+f^{\alpha}-K .
\end{aligned}
$$

Since $\left|f^{\alpha}\right| \leq K$ and by suitably applying Young's inequality: $2 a b \leq r a^{2}+\frac{b^{2}}{r}$, it is clear that there exist $\kappa$ depending only on $N_{2}$ such that the right hand side of the last inequality is negative. So, for this choice of $\kappa$ we have

$$
\delta_{\tau}^{s_{0}} \psi+\sup _{\alpha \in a}\left[\mathcal{L}_{h_{1}}^{\alpha} \psi+f^{\alpha}+\sum_{p} k_{p}\left[\psi\left(t, x+\eta^{\alpha}(x, p)\right)-\psi(t, x)\right]\right] \leq 0
$$

and

$$
\begin{aligned}
\psi\left(s_{0}, x\right) & =L\left(\gamma\left|x-x_{0}\right|^{2}+\gamma^{-1}\right)+v_{\tau, h}\left(s_{0}, x_{0}\right) \\
& \geq L\left|x-x_{0}\right|+v_{\tau, h}\left(s_{0}, x_{0}\right) \geq v_{\tau, h}\left(s_{0}, x\right) .
\end{aligned}
$$

We now apply Lemma 3.2 on $M_{s_{0}}$ and conclude that

$$
v_{\tau, h}\left(t, x_{0}\right) \leq \psi\left(t, x_{0}\right)=\gamma L \kappa\left(s_{0}-t\right)+\gamma^{-1} L+K\left(s_{0}-t\right)+v_{\tau, h}\left(s_{0}, x_{0}\right) .
$$

Minimizing with respect to the $\gamma>0$ and using the fact $\left(s_{0}-t\right) \leq 1$, we conclude

$$
\begin{aligned}
v_{\tau, h}\left(t, x_{0}\right)-v_{\tau, h}\left(s_{0}, x_{0}\right) & \leq 2 L \kappa^{\frac{1}{2}}\left|s_{0}-t\right|^{\frac{1}{2}}+K s_{0}^{\frac{1}{2}}\left|s_{0}-t\right|^{\frac{1}{2}} \\
& \leq N\left(d_{1}, T, K, \nu(E)\right)(L+1)\left|s_{0}-t\right|^{\frac{1}{2}} .
\end{aligned}
$$

The estimate for the other side is obtained similarly.

We close this section by giving the proof of Theorem 3.4

Lemma 4.7. Assume (A.1), (A.2), (A.3), (3.1), (3.2), $h_{1}, h_{2}, \tau \leq K$, and

$$
L:=\sup _{(t, x),(t, y) \in \bar{Q}_{T}, x \neq y} \frac{\left|v_{\tau, h}(t, x)-v_{\tau, h}(t, y)\right|}{|x-y|} \text {. }
$$

Then we have

$$
\left|v_{\tau, h}(s, x)-v_{\tau, h}(t, x)\right| \leq N(1+L)\left(|s-t|^{\frac{1}{2}}+\tau^{\frac{1}{2}}\right),
$$

where $N$ depends only on $K, d_{1}$ and $\nu(E)$.

Proof. If $|t-s| \geq 1$, then $\left|v_{\tau, h}(t, x)-v_{\tau, h}(s, x)\right| \leq 2\left|v_{\tau, h}\right|_{0}|t-s|^{\frac{1}{2}}$. We may therefore assume $|t-s| \leq 1$.

Assume (without loss of generality) that $s-t=n \tau+\gamma$ where $\gamma \in[0, \tau)$ and $n$ is a natural number. If $\gamma=0$, then we apply Lemma 4.6 on $(t, 0)+\mathcal{M}_{n \tau}$ and conclude

$$
\left|v_{\tau, h}(t, x)-v_{\tau, h}(t+n \tau, x)\right| \leq N|n \tau|^{\frac{1}{2}} .
$$

Now, for other case when $0<\gamma<\tau$ we have,

$$
\begin{aligned}
\left|v_{\tau, h}(t, x)-v_{\tau, h}(s, x)\right| & \leq\left|v_{\tau, h}(t, x)-v_{\tau, h}(t+n \tau, x)\right|+\left|v_{\tau, h}(s-\gamma, x)-v_{\tau, h}(s, x)\right| \\
& \leq N|n \tau|^{\frac{1}{2}}+\left|v_{\tau, h}(s-\gamma, x)-v_{\tau, h}(s, x)\right| .
\end{aligned}
$$


Therefore, we have to estimate $\left|v_{\tau, h}(s-\gamma, x)-v_{\tau, h}(s, x)\right|$ for $\gamma \in(0, \tau)$ and $s-\gamma \geq 0$. Define the following functions on $(s, 0)+\overline{\mathcal{M}}_{T-s}$ :

$$
\left[\hat{\sigma}_{k}^{\alpha}, \hat{b}_{k}^{\alpha}, \hat{c}^{\alpha}, \hat{f}^{\alpha}\right](r, y)=\left[\sigma_{k}^{\alpha}, b_{k}^{\alpha}, c^{\alpha}, f^{\alpha}\right](r-\gamma, y)
$$

and

$$
u=v_{\tau, h} ; \hat{u}(r, y)=v_{\tau, h}(r-\gamma, y),
$$

for all $(r, y) \in(s, 0)+\overline{\mathcal{M}}_{T-s}$. Then, on $(s, 0)+\overline{\mathcal{M}}_{T-s}, \hat{u}$ satisfies 3.3 constructed from $\hat{\sigma}_{k}^{\alpha}, \hat{b}_{k}^{\alpha}, \hat{c}^{\hat{\alpha}}, \hat{f}^{\alpha}$ and unchanged jump amplitudes $\eta^{\alpha}$. Noticing the fact that the parameter $\epsilon$ in Theorem 4.4 after using (A.3) is less than $N \gamma^{\frac{1}{2}}$ and also using the $x$-Lipschitz continuity of $v_{\tau, h}$ we have, after applying Theorem 4.4 on $(s, 0)+\overline{\mathcal{M}}_{T-s}$,

$$
\begin{aligned}
\left|v_{\tau, h}(s, x)-v_{\tau, h}(s-\gamma, y)\right| & =|u(s, x)-\hat{u}(s, y)| \\
& \leq N \gamma^{\frac{1}{2}}+\sup _{y \in \mathbb{R}^{d}}|u(T, y)-\hat{u}(T, y)| \\
& =N \gamma^{\frac{1}{2}}+\left|v_{\tau, h}(T, x)-v_{\tau, h}(T-\gamma, y)\right| .
\end{aligned}
$$

Lastly, we are left with estimating $\left|v_{\tau, h}(T, x)-v_{\tau, h}(T-\gamma, y)\right|$. To this end, consider the grid $\mathcal{M}_{\tau}$. With a slight abuse of the notation, we define $\hat{u}(r, x)$ on $\overline{\mathcal{M}}_{\tau}$ by

$$
\hat{u}(0, x)=v_{\tau, h}(T-\gamma, x) ; \hat{u}(\tau, x)=v_{\tau, h}(T, x) .
$$

Then $\hat{u}$ solves (3.3) on $\mathcal{M}_{\tau}$, with an obvious shift (by a quantity $t-\gamma$ in the backward direction) in the time variable of the coefficients, and therefore by Lemma 4.6 we must have

$$
|\hat{u}(\tau, x)-\hat{u}(0, x)| \leq N \tau^{\frac{1}{2}},
$$

i.e.,

$$
\left|v_{\tau, h}(T, x)-v_{\tau, h}(T-\gamma, x)\right| \leq N \tau^{\frac{1}{2}}
$$

Finally, we conclude by combining the estimates 4.28, 4.29), and 4.30.

Proof of Theorem 3.4. Estimates (3.8), 3.9) follow from Theorem 4.4, and estimate 3.10 from Lemma 4.7 if $\tau$ is small enough (then $L<\infty$ by Theorem 4.3).

\section{Singular Lévy measures and optimal error bounds in a SPecial Case}

In this section we address the case of unbounded (singular) Lévy measures. Specifically, in a special case, we introduce a modified difference-quadrature scheme for which we obtain an optimal (under our assumptions) convergence rate.

In general the Lévy measure need not be bounded and/or compactly supported, but it always satisfies the condition

$$
\int_{|z| \leq 1}|z|^{2} \nu(d z)+\int_{|z|>1} e^{K|z|} \nu(d z)<\infty
$$

for some constant $K \geq 0$. Under this condition, the jump amplitude $\eta^{\alpha}$ must satisfy

$$
\left|\eta^{\alpha}(x, z)\right|+\sup _{|h|>0} \frac{1}{h}\left|\eta^{\alpha}(x+h, z)-\eta^{\alpha}(x, z)\right| \leq N\left(|z| 1_{|z| \leq 1}+e^{K|z|} 1_{|z|>1}\right) .
$$

Conditions (5.1) and (5.2) along with (A.1) and (A.3) ensure that the underlying stochastic control problem is well-defined. Moreover, the initial value problem (1.1) and 1.2 , with $\mathcal{I}$ defined in 1.3 , possesses a unique Hölder continuous viscosity 
solution. We refer to [16] for the proof of this result and for the precise definition of viscosity solutions in this setting.

To solve such problems numerically the first step is often (see, e.g., [12, 18) to approximate the Lévy measure $\nu$ by a finite and compactly supported measure of the form $\nu_{r, R}(z)=\mathbf{1}_{r<|z|<R} \nu(d z)$ (occasionally one also adds a diffusion term to the equation to account for the small jumps $|z| \leq r$ ), and then to discretize the corresponding Bellman equation by a finite difference-quadrature scheme like (3.3) of Section 3 The truncation error related to $r, R$ can be estimated following the arguments of [18, while for a fixed truncation level, i.e., for a fixed choice of $r$ and $R$, the error coming from the numerical scheme is given by Theorem 3.5 .

By choosing $r, R$ optimally in terms of $\tau$ and $h$, it should, at least in in principle, be possible to derive a convergence rate for this scheme. However, this is not straightforward since the error bound of Theorem 3.5 only holds if either $\lambda$ is sufficiently large or $\tau$ is sufficiently small when $r$ is small (and $\nu_{r, R}(E)$ is large), see the proofs of Theorems 3.5 and 4.3 . This difficulty can most likely be overcome, for example, by an iteration argument of the type used to prove the Hölder estimates in [4. Perhaps more important, such a convergence rate cannot be optimal because the Lipschitz estimate in Theorem 4.3 is not optimal when the Lévy measure is singular (it "deteriorates" as $r \rightarrow 0$ ).

In the remaining part of this section we will present a different approach, which in the end will produce a better convergence rate. This approach is based on a direct discretization of the integral term (1.3) (no truncation) and obtaining an optimal Lipschitz estimates for the corresponding numerical scheme. The main idea is to use a more refined Bernstein argument than the one used to prove Theorem 4.3 . one that mimics the Bernstein argument for the continuous integro-PDE (with an singular Lévy measure). In implementing this idea we shall restrict ourselves to the case where the jumps do not depend on $x$ :

$$
\left.\eta^{\alpha}(x, z)=\eta^{\alpha}(z) \quad \text { (i.e., } \eta^{\alpha} \text { does not depend on } x\right) \text {. }
$$

This assumption (along with previous ones) are sufficient to imply a Lipschitz estimate for our modified numerical scheme that applies when the Lévy measure is singular (see below). To obtain a convergence rate for our scheme, we will impose an additional technical condition saying that for a fixed $\alpha$ it is possible to jump only in one direction, i.e.,

$$
\eta^{\alpha}(x, z)=\xi^{\alpha} \eta^{\alpha}(z)
$$

for some direction $\xi^{\alpha} \in \mathbb{R}^{N},\left|\xi^{\alpha}\right|=1$, and a scalar function $\eta^{\alpha}(z)$ satisfying (5.2). This restrictive assumption is used to ensure that the integrand in the unbounded Lévy case is Lipschitz continuous in $z$ (details are given below).

Let us now turn to the precise definition our scheme. To this end, we introduce the finite measures

$$
\nu^{\alpha}(d z):=1_{|z| \leq 1}\left|\eta^{\alpha}(z)\right|^{2} \nu(d z)+1_{|z| \geq 1} \nu(d z) .
$$

These positive measures are nonsingular at the origin. Let $I_{h_{2}}^{\alpha}$ be a quadrature rule satisfying

$$
I_{h_{2}}^{\alpha} f=\sum_{p \in h_{2} \mathbb{Z}^{N} \backslash\{0\}} k_{p}^{\alpha} f(p), \quad \text { where } k_{p}^{\alpha} \geq 0,
$$


and

$$
\left|I_{h_{2}}^{\alpha} f-\int_{E} f(z) \nu^{\alpha}(d z)\right| \leq L_{f} \max _{\alpha} \nu^{\alpha}(E) h_{2},
$$

for bounded Lipschitz continuous functions $f(z)$ with Lipschitz constant $L_{f}$.

Conditions 5.4 and 5.5 are satisfied by Riemann sum approximations and NewtonCotes quadratures of order less than 9 like the compound trapezoidal and Simpson rules. Note that by (5.5), $I_{h_{2}} 1=\sum_{p} k_{p}^{\alpha}=\nu^{\alpha}(E)$ and that $\max _{\alpha} \nu^{\alpha}(E)<\infty$ by (5.1) and 5.2.

We discretize the non-local term in (1.1) in the following way:

$$
\begin{aligned}
& \mathcal{I}_{h_{2}}^{\alpha} u(t, x):= \\
& I_{h_{2}}^{\alpha}\left[\frac{u\left(t, x+\xi^{\alpha} \eta^{\alpha}(z)\right)-u(t, x)+1_{|z| \leq 1} \frac{1}{h}\left(u\left(t, x-h_{1} \xi^{\alpha} \eta^{\alpha}(z)\right)-u(t, x)\right)}{1_{|z| \leq 1}\left|\eta^{\alpha}(z)\right|^{2}+1_{|z|>1}} 1_{\left\{\eta^{\alpha} \neq 0\right\}}\right] .
\end{aligned}
$$

Our new difference-quadrature scheme for (1.1) now reads

$$
\delta_{\tau}^{T} u(t, x)+\sup _{\alpha \in \mathcal{A}}\left[\mathcal{L}_{h_{1}}^{\alpha}(t, x) u+f^{\alpha}(t, x)+\mathcal{I}_{h_{2}}^{\alpha} u\right]=0 .
$$

Existence, uniqueness, and comparison results for (5.6) follow along the same lines as before. Furthermore, we claim that the Lipschitz estimate of Theorem 3.4 is satisfied for (5.6) with the modification that the constant $\tau_{0}$ along with the different constants $N$ appearing in (3.8), (3.9), 3.10) are independent of $\nu(E)$. To verify this claim, it is enough to show that Theorem 4.3 can be proved with $N$ in (4.4) chosen independent of $\nu(E)$.

The " $\nu(E)$ - relevant" term in the proof of Theorem 4.3 is $v_{r}^{-} I_{5 r}$ which in the present context equals $v_{r}^{-} \mathcal{I}_{h_{2}}^{\alpha} v_{r}^{-}$. With a slight abuse of the notation we define

$$
I_{h} w(x):=w(x+\eta(z))-w(x)+1_{|z| \leq 1} \frac{1}{h}{ }_{1}\left[w\left(x-h_{1} \eta^{\alpha}(z)\right)-w(x)\right],
$$

for bounded functions $w(x)$. If, at a point $x, w(x) \leq 0$, then

$$
\begin{aligned}
I_{h} w(x) & :=w(x+\eta(z))-w(x)+1_{|z| \leq 1} \frac{1}{h_{1}}\left[w\left(x-h_{1} \eta^{\alpha}(z)\right)-w(x)\right] \\
& \geq-w^{-}(x+\eta(z))+w^{-}-1_{|z| \leq 1} \frac{1}{h_{1}}\left[w^{-}\left(x-h_{1} \eta^{\alpha}(z)\right)-w^{-}(x)\right] \\
& =-I_{h}\left(w^{-}\right)(x) .
\end{aligned}
$$

Since $(t, x)$ is a maximum point of $V$ (remember that we are following the proof of Theorem 4.3), we have

$$
\begin{aligned}
0 & \geq I_{h} \sum_{r}\left(v_{r}^{-}\right)^{2}-\delta I_{h} C(x) \\
& =2 v_{r}^{-} I_{h} v_{r}^{-}+\text {"positive quantity" }-\delta I_{h} C(x),
\end{aligned}
$$

and, since the sum over $r$ could be restricted to those $r$ for which $v_{r} \leq 0$, it follows from 5.7 that

$$
0 \geq-2 v_{r}^{-} I_{h} v_{r}-\delta I_{h} C(x) .
$$

Hence, by the obvious relation between $I_{h_{2}}$ and $\mathcal{I}_{h_{2}}^{\alpha}$,

$$
v_{r}^{-} I_{5 r}=v_{r}^{-} \mathcal{I}_{h_{2}}^{\alpha} v_{r}^{-} \geq-\frac{\delta}{2} \mathcal{I}_{h_{2}}^{\alpha} C(x) \geq-\frac{\delta}{2} N
$$


where $N$ does not depend on $\nu(E)$. This proves the claim.

For any smooth function $w$, let us define the function

$$
G(z)=\frac{w\left(x+\xi^{\alpha} \eta^{\alpha}(z)\right)-w(x)-\xi^{\alpha} \eta^{\alpha}(z) D_{x} w}{\left|\eta^{\alpha}(z)\right|^{2}}
$$

and observe that

$$
\left|D_{z} G\right| \leq 2\left|\left(\eta^{\alpha}\right)^{\prime}\right|_{0}\left|D_{x}^{3} w\right|_{0},
$$

where $\left(\eta^{\alpha}\right)^{\prime}$ is the $z$-derivative of $\eta^{\alpha}$. Condition $\left.\sqrt{5.3}\right)$ is used to enure the validity of this estimate; For more general forms of $\eta^{\alpha}(z)$ it may not be true. As a consequence of this bound and a split of the domain of integration into the two regions $\{|z| \leq 1\}$ and $\{|z|>1\}$, we obtain the error bound

$$
\left|\mathcal{I}^{\alpha} w-\mathcal{I}_{h}^{\alpha} w\right| \leq N\left(\left(\left|D_{x}^{3} w\right|_{0}+\left|D_{x} w\right|_{0}\right) h_{2}+\left|D_{x}^{2} w\right|_{0} h_{1}\right) .
$$

Denote by $v_{\tau, h}$ the unique solution of $(5.6),(1.2)$ and by $v$ the unique viscosity solution of (1.1), 1.2). In view of (5.8) and the discussion above, if we repeat the proof of Theorem 3.5 we will eventually find that

$$
\left|v-v_{\tau, h}\right| \leq N \min _{\epsilon}\left(\epsilon+h_{2}\left(\frac{1}{\epsilon}+\frac{1}{\epsilon^{2}}\right)+\frac{h_{1}}{\epsilon}+\frac{\tau+h_{1}^{2}}{\epsilon^{3}}\right) \leq N\left(\tau^{\frac{1}{4}}+h_{1}^{\frac{1}{2}}+h_{2}^{\frac{1}{3}}\right),
$$

for sufficiently small $\tau, h_{1}, h_{2}$.

Summarizing, we have proved

Theorem 5.1. Assume that (A.1), (A.3), (5.1), (5.2), (5.3), (5.4), (5.5) hold. Let $v$ and $v_{\tau, h}$ be the solutions of (1.1), (1.2) and (5.6), (1.2), respectively. Then there exists a constant $N$, depending only on $d, d_{1}$, and $K$, such that

$$
\left|v-v_{\tau, h}\right| \leq N\left(\tau^{\frac{1}{4}}+h_{1}^{\frac{1}{2}}+h_{2}^{\frac{1}{3}}\right) .
$$

Remark 5.1. Theorem 5.1 appears to be the first result on convergence rates for numerical schemes of Bellman equations with singular Lévy measures. Note that the convergence rate in Theorem 5.1 does not depend on the strength of the singularity at $z=0$ of the Lévy measure. Furthermore, the result is most likely optimal under the current conditions. However, if we have further information about $\nu$, e.g., if $\nu(d z) \leq N|z|^{-\gamma-M} d z$ in a neighborhood of $z=0$ for some $\gamma \in(0,2)$, then the rate $1 / 3$ can be improved. We leave this to the interested reader.

\section{REFERENCES}

[1] O. Alvarez and A. Tourin. Viscosity solutions of nonlinear integro-differential equations. Ann. Inst. H. Poincaré Anal. Non Linéaire, 13(3):293-317, 1996.

[2] A. L. Amadori. Nonlinear integro-differential evolution problems arising in option pricing: a viscosity solutions approach. Differential Integral Equations, 16(7):787-811, 2003.

[3] G. Barles, R. Buckdahn, and E. Pardoux. Backward stochastic differential equations and integral-partial differential equations. Stochastics Stochastics Rep., 60(1-2):57-83, 1997.

[4] G. Barles and E. R. Jakobsen. On the convergence rate of approximation schemes for Hamilton-Jacobi-Bellman equations. M2AN Math. Model. Numer. Anal., 36(1):33-54, 2002.

[5] G. Barles and E. R. Jakobsen. Error bounds for monotone approximation schemes for Hamilton-Jacobi-Bellman equations. SIAM J. Numer. Anal., 43(2):540-558 (electronic), 2005.

[6] G. Barles and E. R. Jakobsen. Error bounds for monotone approximation schemes for parabolic Hamilton-Jacobi-Bellman equations. Math. Comp.,76(240):1861-1893, 2007.

[7] G. Barles and P. E. Souganidis. Convergence of approximation schemes for fully nonlinear second order equations. Asymptotic Anal., 4(3):271-283, 1991. 
[8] F. E. Benth, K. H. Karlsen, and K. Reikvam. Optimal portfolio management rules in a nonGaussian market with durability and intertemporal substitution. Finance Stoch., 5(4):447467, 2001.

[9] F. E. Benth, K. H. Karlsen, and K. Reikvam. Optimal portfolio selection with consumption and nonlinear integrodifferential equations with gradient constraint: a viscosity solution approach. Finance and Stochastic, 5:275-303, 2001.

[10] F. E. Benth, K. H. Karlsen, and K. Reikvam. Portfolio optimization in a Lévy market with intertemporal substitution and transaction costs. Stoch. Stoch. Rep., 74(3-4):517-569, 2002.

[11] I. H. Biswas, E. R. Jakobsen, and K. H. Karlsen. Error estimates for finite differencequadrature schemes for a class of nonlocal Bellman equations with variable diffusion. Contemp. Math., volume 429, Amer. Math. Soc., pages 19-31, 2007.

[12] R. Cont and P. Tankov. Finacial modeling with jump processes. Chapman \& Hall/CRC, Boca Raton, FL, 2004.

[13] M. G. Crandall, H. Ishii, and P.-L. Lions. User's guide to viscosity solutions of second order partial differential equations. Bull. Amer. Math. Soc. (N.S.), 27(1):1-67, 1992.

[14] W. H. Fleming and H. M. Soner. Controlled Markov processes and viscosity solutions. Springer-Verlag, New York, 1993.

[15] E. R. Jakobsen. On the rate of convergence of approximation schemes for Bellman equations associated with optimal stopping time problems. Math. Models Methods Appl. Sci., 13(5):613$644,2003$.

[16] E. R. Jakobsen and K. H. Karlsen. Continuous dependence estimates for viscosity solutions of integro-PDEs. J. Differential Equations, 212(2):278-318, 2005.

[17] E. R. Jakobsen and K. H. Karlsen. A "maximum principle for semicontinuous functions" applicable to integro-partial differential equations. NoDEA Nonlinear Differential Equations Appl., 13:137-165, 2006.

[18] E. R. Jakobsen, K. H. Karlsen, and C. L. Chioma. Error estimates for approximate solutions to Bellman equations associated with controlled jump-diffusions. Submitted to Numer. Math., 2005.

[19] N. V. Krylov. On the rate of convergence of finite-difference approximations for Bellman's equations. Algebra i Analiz, 9(3):245-256, 1997.

[20] N. V. Krylov. On the rate of convergence of finite-difference approximations for Bellman's equations with variable coefficients. Probab. Theory Related Fields, 117(1):1-16, 2000.

[21] N. V. Krylov. The rate of convergence of finite-difference approximations for Bellman equations with Lipschitz coefficients. Appl. Math. Optim., 52(3):365-399, 2005.

[22] R. Mikulyavichyus and G. Pragarauskas. Nonlinear potentials of the Cauchy-Dirichlet problem for the Bellman integro-differential equation. Liet. Mat. Rink., 36(2):178-218, 1996.

[23] H. Pham. Optimal stopping of controlled jump diffusion processes: a viscosity solution approach. J. Math. Systems Estim. Control, 8(1):27 pp. (electronic), 1998.

(Imran H. Biswas)

Centre of Mathematics for Applications, University of Oslo, P.O. Box 1053, Blindern, NO-0316 OSLO, NORWAY

E-mail address: i.h.biswas@cma.uio.no

(Espen R. Jakobsen)

Norwegian University of Science and Technology, N-7491, Trondheim, Norway

E-mail address: erj@math.ntnu.no

$U R L:$ www.math.ntnu.no/ ${ }^{\sim}$ erj/

(Kenneth Hvistendahl Karlsen)

Centre of Mathematics for Applications, University of Oslo, P.O. Box 1053, Blindern, NO-0316 OSLO, NoRWAy

E-mail address: kennethk@math.uio.no

$U R L$ : folk.uio.no/kennethk/ 